\title{
Think different: applying the old macintosh mantra to the computability of the SUSY auxiliary field problem
}

\author{
Mathew Calkins, ${ }^{a}$ D.E.A. Gates, ${ }^{a}$ S. James Gates Jr. ${ }^{a}$ and William M. Golding ${ }^{b}$ \\ ${ }^{a}$ Center for String and Particle Theory, Department of Physics, University of Maryland, \\ College Park, MD 20742-4111 U.S.A. \\ ${ }^{b}$ Sensors and Electron Devices Directorate, US Army Research Laboratory, \\ Adelphi, Maryland 20783 U.S.A. \\ E-mail: mathewpcalkins@gmail.com, deagates@terpmail.umd.edu, \\ gatess@wam.umd.edu, william.m.golding2 .civ@mail.mil
}

ABSTRACT: Starting with valise supermultiplets obtained from 0-branes plus field redefinitions, valise adinkra networks, and the "Garden Algebra," we discuss an architecture for algorithms that (starting from on-shell theories and, through a well-defined computation procedure), search for off-shell completions. We show in one dimension how to directly attack the notorious "off-shell auxiliary field" problem of supersymmetry with algorithms in the adinkra network-world formulation.

Keywords: Space-Time Symmetries, Gauge Symmetry, Global Symmetries

ARXIV EPRINT: 1502.04164 


\section{Contents}

1 Introduction 1

2 Review of the Siegel-Roček theorem 3

3 In the world of 0-brane valise supermultiplets 3

4 A 0-brane-world formulation of the off-shell SUSY auxiliary field problem

5 Within the world of adinkra network valise supermultiplets $\quad 8$

$\begin{array}{llr}5.1 & \text { Chiral supermultiplet adinkra network valise off-shell } & 9\end{array}$

$\begin{array}{ll}\text { 5.2 Chiral supermultiplet adinkra network valise on-shell } & 10\end{array}$

$\begin{array}{lll}5.3 & \text { Vector supermultiplet adinkra network valise off-shell } & 11\end{array}$

$\begin{array}{lll}5.4 & \text { Vector supermultiplet adinkra network valise on-shell } & 11\end{array}$

6 An adinkra network-world formulation of the off-shell SUSY auxiliary field problem 12

7 On-shell adinkra network deformations $\quad 13$

$\begin{array}{lll}7.1 & \text { On-shell chiral valise matrix deformation } & 15\end{array}$

$\begin{array}{lll}7.2 & \text { On-shell vector valise matrix deformation } & 18\end{array}$

8 The general cryptographic problem analogy to the adinkra network aux$\begin{array}{lr}\text { iliary field problem } & \mathbf{2 0}\end{array}$

9 Summary and conclusion $\quad 21$

\section{Introduction}

In 1979, one of the authors (SJG) was invited to the California Institute of Technology by Dr. J.H. Schwarz for a program of study on the issue of finding a set of auxiliary fields with which to close the supersymmetry algebra on the component fields of the $10 \mathrm{D}, \mathcal{N}=1$ Maxwell vector supermultiplet without the use of equations of motion. The study was not completely satisfactory as no set of such fields were identified. This situation has remains unchanged.

Later in 1981, there was formulated a "No-Go" theorem [1] which apparently explained the result of the earlier study. The abstract to this paper by Siegel and Roček that presented the theorem read 
Applying a simple counting argument to all supermultiplets, we find that for the $\mathcal{N}=4$ super Yang-Mills theory the auxiliary field problem cannot have a solution within any previously known framework. We propose alternatives.

Since the $4 \mathrm{D}, \mathcal{N}=4$ Maxwell vector supermultiplet is related to the $10 \mathrm{D}, \mathcal{N}=1$ version via torus compactification, the study result would seem covered.

While this argument is simple and elegant, it has at least one puzzling aspect.

It is widely accepted that the $10 \mathrm{D}, \mathcal{N}=1$ and $4 \mathrm{D}, \mathcal{N}=4$ Maxwell supermultiplets can be embedded within a formulation involving unconstrained super $p$-forms. This fact should imply the existence of some type of off-shell formulation containing the fields of the on-shell theory. Based on this superspace argument there should exist an off-shell completion of the $4 \mathrm{D}, \mathcal{N}=4$ Maxwell supermultiplet.

It has been known since the work of [2] that the super 1-form formulation of the 10D, $\mathcal{N}=1$ Maxwell and Yang-Mills supermultiplets have a rather distinctive structure in terms of the constraints that describe the theories in comparison to other similar theories. In the $10 \mathrm{D}$ case, there is a spinor-spinor field strength component that vanishes in three, four, and six dimensions [3] is non-zero in ten dimensions. This difference was used in the work of [2] to provide the first superspace description of the lowest order open superstring corrections and has been verified a number of times since (see e.g. [3-7]).

Thus, there exists a contrast between these two widely accepted results. The work in [1] concerns the action (with off-shell supersymmetry), while [2] gives only the field equations (thus on-shell, although with contributions from integrating out higher massive modes from the open superstring. Of course, the resolution of the contrast must lie in the fact that some assumption made in one approach does not apply to the other. Knowing this, however, does not provide a detailed explanation.

In 1995, the presence of sets of matrices with certain regularities [8,9] was noted to occur (presumably) in all 1D systems that realize supersymmetry in a linear manner. The matrices (given the designation of L-matrices and R-matrices) would later become recognized as the adjacency matrices of adinkra networks [10]. This latter identification became critical in providing a definition of these matrices independent of field theory models and opening a path to totally unexpected connections to subjects such as cubical cohomology [11], error-correcting codes [12-14], ranked poset [15], Coxeter Groups [17] and most recently Riemann surfaces [18].

Soon after our discovery of the ubiquity of L-matrices and R-matrices in 1D SUSY theories, we proposed [19-21] that these might play a vital role in attacking the off-shell SUSY auxiliary field problem via a technique given the acronym of RADIO. It was envisioned that this technique permits the derivation of new on-shell and off-shell representations by starting from a D-dimensional, $\mathcal{N}$-extended theory. The steps of the process begin by reducing $(\mathrm{R})$ the higher dimensional theory to $1 \mathrm{D}$, followed by performing certain "automorphic duality" (AD) transformations, next integrating additional 1D representations (I) and then oxidizing (O) back up to the higher dimensional spacetime. The actions of $(\mathrm{R})$ and $(\mathrm{AD})$ together produce what we now refer to as "valise supermultiplets." These provide the starting point in this current work. The main focus of this work will be to 
indicate how the (I) can be carried out. We discuss the general philosophy of this step and also show by explicit calculation how this is done.

\section{Review of the Siegel-Roček theorem}

During 2009 in exchanges between M. Faux and SJG, the following discussion for understanding the essential points of the Siegel-Roček argument were noted.

The smallest off-shell $\mathcal{N}$-extended supermultiplets in four-dimensions have $2^{2 N-1}$ component fermions and the same number of component bosons. The number of off-shell component fermions $F$ in any non-minimal $4 \mathrm{D}$ supermultiplet must be an integer multiple of the component fermions in the minimal multiplet. Thus, $F=2^{2 N-1} \mathrm{~m}$, where $\mathrm{m}$ is some positive integer.

All fermions carry an odd number of $\mathrm{SO}(\mathrm{N})$ fundamental indices. As a result, all fermions carry an integer multiple of $4 \mathcal{N}$ off-shell degrees of freedom, where the 4 reflects the dimensionality of a minimal spinor.

A given off-shell multiplet has $f$ fermionic degrees of freedom corresponding to propagating degrees of freedom, plus some number of auxiliary fermions. Auxiliary fermions come paired. It follows that $F=f+2(4 \mathcal{N}) n$, where $n$ counts the number of auxiliary fermion reduced pairings. Thus, $n$ counts the number of minimal spinors assembled to form a given auxiliary fermion representation.

By comparing the two restrictions on the number of fermion components, we conclude $f+8 \mathcal{N} n=2^{2 \mathcal{N}-1} m$. Adapting this to the $4 \mathrm{D}$ cases $\mathcal{N}=2$ and $\mathcal{N}=4$, this yields

$$
\begin{aligned}
& \mathcal{N}=2: f+16 n=8 m, \\
& \mathcal{N}=4: f+32 n=128 m .
\end{aligned}
$$

For the cases of the $\mathcal{N}=2$ vector and tensor supermultiplets, we have $f=8, n=0$, and $m=1$. For the case of the $\mathcal{N}=4$ vector supermultiplet, we have $f=16$, since there are four physical fermions transforming as a 4 under $\mathrm{SO}(4)$. We can then rearrange the second equation above to read $n=4 m-\frac{1}{2}$. This equation has no solutions for integer $m$ and integer $n$.

\section{In the world of 0-brane valise supermultiplets}

All our previous explorations suggests that via the (R) and (AD) steps of the RADIO proposal, any linear representation of spacetime supersymmetry can be made to depend on a single real parameter in the forward light-cone [22]. Under field redefinition using derivatives or integrals, such representations can be brought to a universal form of a valise supermultiplet

$$
\mathrm{D}_{a} \Phi_{\Lambda}=i\left(\mathrm{~L}_{\Lambda}\right)_{a}{ }^{\widehat{\Lambda}} \Psi_{\widehat{\Lambda}}, \quad \mathrm{D}_{a} \Psi_{\widehat{\Lambda}}=\left(\mathrm{R}^{\Lambda}\right)_{\widehat{\Lambda} a} \partial_{\tau} \Phi_{\Lambda}
$$

Here the explicit forms of the constants $\left(\mathrm{L}_{\Lambda}\right){ }^{\widehat{\Lambda}}$ and $\left(\mathrm{R}^{\Lambda}\right)_{\widehat{\Lambda}} a$, as well as the field variables $\Phi_{\Lambda}$, and $\Psi_{\widehat{\Lambda}}$ vary from supermultiplet to supermultiplet. The condition that the field 
variables in (3.1) form representations of spacetime supersymmetry just takes the simplified form

$$
\left\{\mathrm{D}_{a}, \mathrm{D}_{b}\right\}=i 2\left(\gamma^{0}\right)_{a b} \partial_{\tau},
$$

when calculated on any of the component fields from any of the supermultiplets.

Implementing the $(\mathrm{R})$ and $(\mathrm{AD})$ parts of the RADIO proposal necessarily breaks $\mathrm{SO}(1,3)$ covariance. However, as noted in [23], in place of the $\mathrm{SO}(1,3)$ symmetry a new $\mathrm{SU}(2) \otimes \mathrm{SU}(2)$ symmetry appears in the equations that emerge for off-shell valise supermultiplets. The generators of these two commuting $\mathrm{SU}(2)$ symmetries are given by

$$
i \frac{1}{4}\left[\gamma^{m}, \gamma^{n}\right]
$$

for the generator of purely spatial rotations and

$$
i \gamma^{0}, \quad \gamma^{5}, \quad \gamma^{0} \gamma^{5}
$$

for the generators of an extended SU(2) R-symmetry [24].

To make this more concrete, we illustrate some familiar representations after application of the (R) and (AD) steps and obtain the results of [23]:

(a.) Chiral Supermultiplet (CS);

$$
\begin{gathered}
\mathrm{D}_{a} A=\psi_{a}, \quad \mathrm{D}_{a} B=i\left(\gamma^{5}\right)_{a}{ }^{b} \psi_{b}, \quad \mathrm{D}_{a} F=\left(\gamma^{0}\right)_{a}{ }^{b} \psi_{b}, \quad \mathrm{D}_{a} G=i\left(\gamma^{5} \gamma^{0}\right)_{a}{ }^{b} \psi_{b}, \\
\mathrm{D}_{a} \psi_{b}=i\left(\gamma^{0}\right)_{a b}\left(\partial_{\tau} A\right)-\left(\gamma^{5} \gamma^{0}\right)_{a b}\left(\partial_{\tau} B\right)-i C_{a b}\left(\partial_{\tau} F\right)+\left(\gamma^{5}\right)_{a b}\left(\partial_{\tau} G\right),
\end{gathered}
$$

(b.) Vector Supermultiplet (VS);

$$
\begin{aligned}
\mathrm{D}_{a} A_{m} & =\left(\gamma_{m}\right)_{a}{ }^{b} \lambda_{b}, \quad \mathrm{D}_{a} \mathrm{~d}=i\left(\gamma^{5} \gamma^{0}\right)_{a}{ }^{b} \lambda_{b}, \\
\mathrm{D}_{a} \lambda_{b} & =-i\left(\gamma^{0} \gamma^{m}\right)_{a b}\left(\partial_{\tau} A_{m}\right)+\left(\gamma^{5}\right)_{a b}\left(\partial_{\tau} \mathrm{d}\right),
\end{aligned}
$$

(c.) Tensor Supermultiplet (TS);

$$
\begin{aligned}
\mathrm{D}_{a} \varphi & =\chi_{a}, \quad \mathrm{D}_{a} B_{m n}=-\frac{1}{4}\left(\left[\gamma_{m}, \gamma_{n}\right]\right)_{a}{ }^{b} \chi_{b} \\
\mathrm{D}_{a} \chi_{b} & =i\left(\gamma^{0}\right)_{a b} \partial_{\tau} \varphi-i \frac{1}{2}\left(\gamma^{0}\left[\gamma^{m}, \gamma^{n}\right]\right)_{a b} \partial_{\tau} B_{m n}
\end{aligned}
$$

(d.) Axial vector Supermultiplet (AVS);

$$
\begin{aligned}
\mathrm{D}_{a} U_{m} & =i\left(\gamma^{5} \gamma_{m}\right)_{a}{ }^{b} \widetilde{\lambda}_{b}, \quad \mathrm{D}_{a} \widetilde{\mathrm{d}}=-\left(\gamma^{0}\right)_{a}{ }^{b} \partial_{\tau} \widetilde{\lambda}_{b}, \\
\mathrm{D}_{a} \widetilde{\lambda}_{b} & =\left(\gamma^{5} \gamma^{0} \gamma^{m}\right)_{a b}\left(\partial_{\tau} U_{m}\right)+i C_{a b} \widetilde{\mathrm{d}},
\end{aligned}
$$

(e.) Axial tensor Supermultiplet (ATS); and

$$
\begin{aligned}
\mathrm{D}_{a} \widetilde{\varphi} & =i\left(\gamma^{5}\right)_{a}{ }^{b} \widetilde{\chi}_{b}, \quad \mathrm{D}_{a} \widetilde{B}_{m n}=-i \frac{1}{4}\left(\gamma^{5}\left[\gamma_{m}, \gamma_{n}\right]\right)_{a}{ }^{b} \widetilde{\chi}_{b} \\
\mathrm{D}_{a} \widetilde{\chi}_{b} & =-\left(\gamma^{0} \gamma^{5}\right)_{a b} \partial_{\tau} \widetilde{\varphi}+\frac{1}{2}\left(\gamma^{0} \gamma^{5}\left[\gamma^{m}, \gamma^{n}\right]\right)_{a b} \partial_{\tau} \widetilde{B}_{m n} .
\end{aligned}
$$


(f.) Real Scalar Supermultiplet (RSS);

$$
\begin{aligned}
\mathrm{D}_{a} K & =\zeta_{a}, \mathrm{D}_{a} \mathrm{~d}=-\left(\gamma^{0}\right)_{a}^{d} \Lambda_{d} \\
\mathrm{D}_{a} M & =\frac{1}{2} \Lambda_{a}-\frac{1}{2}\left(\gamma^{0}\right)_{a}^{d} \zeta_{d}, \mathrm{D}_{a} N=-i \frac{1}{2}\left(\gamma^{5}\right)_{a}^{d} \Lambda_{d}+i \frac{1}{2}\left(\gamma^{5} \gamma^{0}\right)_{a}^{d} \zeta_{d} \\
\mathrm{D}_{a} U_{0} & =i \frac{1}{2}\left(\gamma^{5} \gamma_{0}\right)_{a}^{d} \Lambda_{d}-i \frac{1}{2}\left(\gamma^{5}\right)_{a}^{d} \zeta_{d}, \mathrm{D}_{a} U_{m}=i \frac{1}{2}\left(\gamma^{5} \gamma_{m}\right)_{a}^{d} \Lambda_{d}-i \frac{1}{2}\left(\gamma^{5} \gamma^{0} \gamma_{m}\right)_{a}^{d} \zeta_{d} \\
\mathrm{D}_{a} \zeta_{b} & =i\left(\gamma^{0}\right)_{a b} \partial_{\tau} K+\left(\gamma^{5} \gamma^{\mu}\right)_{a b} \partial_{\tau} U_{\mu}+i C_{a b} \partial_{\tau} M+\left(\gamma^{5}\right)_{a b} \partial_{\tau} N \\
\mathrm{D}_{a} \Lambda_{b} & =i\left(\gamma^{0}\right)_{a b} \partial_{\tau} M+\left(\gamma^{5} \gamma^{0}\right)_{a b} \partial_{\tau} N+\left(\gamma^{5} \gamma^{0} \gamma^{\nu}\right)_{a b} \partial_{\tau} U_{\nu}+i C_{a b} \partial_{\tau} \mathrm{d}
\end{aligned}
$$

In particular for each of the supermultiplets, one can define a 'vector' of bosonic (denoted by $\Phi_{\Lambda}$ ) and fermionic (denoted by $\Psi_{\widehat{\Lambda}}$ ) valise supermultiplet variables. In the case of the CS we have

$$
\Phi_{\Lambda}=(A, B, F, G), \quad \Psi_{\widehat{\Lambda}}=\left(\psi_{a}\right)
$$

for the VS we have

$$
\Phi_{\Lambda}=\left(A_{m}, \mathrm{~d}\right), \quad \Psi_{\widehat{\Lambda}}=\left(\lambda_{a}\right)
$$

for the TS we have

$$
\Phi_{\Lambda}=\left(\varphi, B_{m n}\right), \quad \Psi_{\widehat{\Lambda}}=\left(\chi_{a}\right)
$$

for the AVS we have

$$
\Phi_{\Lambda}=\left(U_{m}, \widetilde{\mathrm{d}}\right), \quad \Psi_{\widehat{\Lambda}}=\left(\widetilde{\lambda}_{a}\right)
$$

for the ATS we have

$$
\Phi_{\Lambda}=\left(\widetilde{\varphi}, \widetilde{B}_{m n}\right), \quad \Psi_{\widehat{\Lambda}}=\left(\widetilde{\chi}_{a}\right)
$$

and for the RSS we have

$$
\Phi_{\Lambda}=\left(\mathrm{K}, M, N, U_{0}, U_{m}, \mathrm{~d}\right), \quad \Psi_{\widehat{\Lambda}}=\left(\zeta_{a}, \Lambda_{a}\right) .
$$

As seen above, the $\Lambda$ indices are allowed to range over distinct bosonic representations of $\mathrm{SO}(1,3)$ and similarly the $\widehat{\Lambda}$ indices (in the most general case) are allowed to range over distinct fermionic representations of $\mathrm{SO}(1,3)$.

The explicit forms of the $L_{\Lambda}$ and $R^{\Lambda}$ coefficients can now be read out for each of the supermultiplets. Furthermore, as seen from these examples, the $\mathrm{L}_{\Lambda}$ and $\mathrm{R}^{\Lambda}$ coefficients are constructed from Lorentz invariant tensors, $\gamma$-matrices, and powers thereof. Thus, information about the space-time spin of the fields in the supermultiplets is encoded in these coefficients even though the field variables only depend on time. We conjecture that every linear off-shell representation of supersymmetry can always be subject to 0-brane reduction $(\mathrm{R})$, field redefinitions ( $\mathrm{AD}$, and possibly linearization) such that equations (3.1) and (3.2) are satisfied.

Now in order to focus on the SUSY auxiliary field problem, we concentrate solely on the chiral supermultiplet and vector supermultiplet in the remainder of this section. The 
on-shell version of these two supermultiplets are given below. For the on-shell version of the chiral supermultiplet we have

$$
\begin{aligned}
\mathrm{D}_{a} A & =\psi_{a}, \quad \mathrm{D}_{a} B=i\left(\gamma^{5}\right)_{a}{ }^{b} \psi_{b}, \\
\mathrm{D}_{a} \psi_{b} & =i\left(\gamma^{0}\right)_{a b} \partial_{\tau} A-\left(\gamma^{5} \gamma^{0}\right)_{a b} \partial_{\tau} B,
\end{aligned}
$$

leading to

$$
\begin{gathered}
\left\{\mathrm{D}_{a}, \mathrm{D}_{b}\right\} A=i 2\left(\gamma^{0}\right)_{a b} \partial_{\tau} A, \quad\left\{\mathrm{D}_{a}, \mathrm{D}_{b}\right\} B=i 2\left(\gamma^{0}\right)_{a b} \partial_{\tau} B, \\
\left\{\mathrm{D}_{a}, \mathrm{D}_{b}\right\} \psi_{c}=i 2\left(\gamma^{0}\right)_{a b} \partial_{\tau} \psi_{c}-i\left(\gamma^{\mu}\right)_{a b}\left(\gamma_{\mu} \gamma^{0}\right)_{c}{ }^{d} \partial_{\tau} \psi_{d} .
\end{gathered}
$$

The final term in (3.18) is characteristic of an on-shell theory, an extra term appears relative to the off-shell result shown in (3.2). Note that (3.17) is exactly of the same form as (3.11), but with the important exception that the $F$ and $G$ field variables are deleted. In the on-shell theory, the absence of these two bosonic fields leads to the extra term in the evaluation of the algebra acting on the fermionic field. Going from on-shell to off-shell corresponds by augmenting the bosonic vector from $(A, B)$ to $(A, B, F, G)$ and ensures the condition in (3.2) is satisfied.

The on-shell 0-brane formulation of the vector supermultiplet is given by

$$
\begin{aligned}
\mathrm{D}_{a} A_{m} & =\left(\gamma_{m}\right)_{a}^{b} \lambda_{b}, \\
\mathrm{D}_{a} \lambda_{b} & =-i\left(\gamma^{0} \gamma^{m}\right)_{a b}\left(\partial_{\tau} A_{m}\right),
\end{aligned}
$$

and once again we calculate the anticommutator on the fields

$$
\begin{aligned}
\left\{\mathrm{D}_{a}, \mathrm{D}_{b}\right\} A_{m}= & i 2\left(\gamma^{0}\right)_{a b} \partial_{\tau} A_{m} \\
\left\{\mathrm{D}_{a}, \mathrm{D}_{b}\right\} \lambda_{c}= & i 2\left(\gamma^{0}\right)_{a b} \partial_{\tau} \lambda_{c}-i \frac{1}{2}\left(\gamma^{\mu}\right)_{a b}\left(\gamma_{\mu} \gamma^{0}\right)_{c}{ }^{d} \partial_{\tau} \lambda_{d} \\
& +i \frac{1}{16}\left(\left[\gamma^{\alpha}, \gamma^{\beta}\right]\right)_{a b}\left(\left[\gamma_{\alpha}, \gamma_{\beta}\right] \gamma^{0}\right)_{c}{ }^{d} \partial_{\tau} \lambda_{d}
\end{aligned}
$$

to see the emergence of two extra terms appearing relative to the off-shell' result shown in (3.2). This review has now set the stage for a statement of the off-shell SUSY auxiliary field problem we study in this work. The result in (3.19) is the same as the result in (3.12) with the exception that the latter does not include the $\mathrm{d}$ bosonic field variable. In the on-shell' theory, the absence of the bosonic d field leads to the extra two terms in the evaluation of the algebra acting on the fermionic fields. So going from on-shell to off-shell corresponds to increasing the bosonic vector from $\left(A_{m}\right)$ to $\left(A_{m}, \mathrm{~d}\right)$.

For the 0-brane valise chiral supermultiplet with field content vectors described by (3.11), the commutator algebra (3.2) is satisfied on $\Phi_{\Lambda}$ and on $\Psi_{\widehat{\Lambda}}$. For the 0-brane valise chiral supermultiplet with field content vectors described by (3.17), the commutator algebra (3.18) is satisfied on $\Phi_{\Lambda}$ and on $\Psi_{\widehat{\Lambda}}$. For the 0-brane valise vector supermultiplet with field content vectors described by (3.12), the commutator algebra (3.2) is satisfied on $\Phi_{\Lambda}$ and on $\Psi_{\widehat{\Lambda}}$. For the 0-brane valise vector supermultiplet with field content vectors described by (3.19), the commutator algebra (3.20) is satisfied on $\Phi_{\Lambda}$ and on $\Psi_{\widehat{\Lambda}}$. 


\section{A 0-brane-world formulation of the off-shell SUSY auxiliary field prob- lem}

Let $\Phi_{\Lambda}(\tau)$, and $\Psi_{\widehat{\Lambda}}(\tau)$ denote arbitrary bosonic and fermionic sets of functions. All the bosonic functions satisfy the equation

$$
\Phi_{\Delta}\left(\tau_{1}\right) \Phi_{\Lambda}\left(\tau_{2}\right)=+\Phi_{\Lambda}\left(\tau_{2}\right) \Phi_{\Delta}\left(\tau_{1}\right)
$$

and all the fermionic functions satisfy the equation

$$
\Psi_{\widehat{\Delta}}\left(\tau_{1}\right) \Psi_{\widehat{\Lambda}}\left(\tau_{2}\right)=-\Psi_{\widehat{\Lambda}}\left(\tau_{2}\right) \Psi_{\widehat{\Delta}}\left(\tau_{1}\right)
$$

The off-shell auxiliary field problem then asks that one determine all sets of bosonic functions $\Phi_{\Lambda}(\tau)$, sets of fermionic functions $\Psi_{\widehat{\Lambda}}(\tau)$, constant coefficients $\left(\mathrm{L}_{\Lambda}\right)_{a}{ }^{\widehat{\Lambda}}$, and $\left(\mathrm{R}^{\Lambda}\right)_{\widehat{\Lambda}} a$ (where these coefficients are constructed from Lorentz invariant tensors and gamma matrices) such that the equations

$$
\mathrm{D}_{a} \Phi_{\Lambda}=i\left(\mathrm{~L}_{\Lambda}\right)_{a}{ }^{\widehat{\Lambda}} \Psi_{\widehat{\Lambda}}, \quad \mathrm{D}_{a} \Psi_{\widehat{\Lambda}}=\left(\mathrm{R}^{\Lambda}\right)_{\widehat{\Lambda}}{ }^{a} \partial_{\tau} \Phi_{\Lambda}
$$

necessarily implies

$$
\left\{\mathrm{D}_{a}, \mathrm{D}_{b}\right\}=i 2\left(\gamma^{0}\right)_{a b} \partial_{\tau}
$$

and this should be done in an irreducible manner in the space of field vectors. With the exception of the $4 \mathrm{D}, \mathcal{N}=1$ double tensor multiplet, ${ }^{1}$ there is a solution for (4.1)-(4.4) in the case of every studied supermultiplet known to these authors. The solution to this problem is generally not known for either $\mathcal{N}$-extended supersymmetry or supersymmetry in higher space time dimensions than four.

The most prominent case showing such a failure is the $4 \mathrm{D}, \mathcal{N}=4$ Maxwell Supermultiplet. Here the field content vectors take the respective forms

$$
\Phi_{\Lambda}=\left(A_{m}, A^{\mathcal{I}}, B^{\mathcal{I}}, \mathrm{d}, F^{\mathcal{I}}, G^{\mathcal{I}}\right), \quad \Psi_{\widehat{\Lambda}}=\left(\lambda_{a}, \psi_{a}{ }^{\mathcal{I}}\right)
$$

and these are written appropriately for the realization of one of the four supersymmetries in an off-shell manner. The indices $\mathcal{I}, \mathcal{J}$, etc. here and in the following discussion take on three values. The 0-brane version of an invariant action is given by [25, 26]

$$
\begin{aligned}
\mathcal{L}= & \frac{1}{2}\left(\partial_{\tau} A^{\mathcal{I}}\right)\left(\partial_{\tau} A^{\mathcal{I}}\right)+\frac{1}{2}\left(\partial_{\tau} B^{\mathcal{I}}\right)\left(\partial_{\tau} B^{\mathcal{I}}\right)+\frac{1}{2}\left(\partial_{\tau} F^{\mathcal{I}}\right)\left(\partial_{\tau} F^{\mathcal{I}}\right)+\frac{1}{2}\left(\partial_{\tau} G^{\mathcal{I}}\right)\left(\partial_{\tau} G^{\mathcal{I}}\right) \\
& +\frac{1}{2}\left(\partial_{\tau} A_{m}\right)\left(\partial_{\tau} A_{m}\right)+\frac{1}{2}\left(\partial_{\tau} \mathrm{d}\right)\left(\partial_{\tau} \mathrm{d}\right)+i \frac{1}{2}\left(\gamma^{0}\right)^{a b} \psi_{a}^{\mathcal{I}} \partial_{\tau} \psi_{b}^{\mathcal{I}}+i \frac{1}{2}\left(\gamma^{0}\right)^{c d} \lambda_{c} \partial_{\tau} \lambda_{d}
\end{aligned}
$$

\footnotetext{
${ }^{1}$ See the work in $[23]$ for details.
} 
The four supercharges can be represented by $\mathrm{D}_{a}$ and $\mathrm{D}_{a}^{\mathcal{I}}$ where

$$
\begin{aligned}
\mathrm{D}_{a} A^{\mathcal{J}}= & \psi_{a}^{\mathcal{J}}, \quad \mathrm{D}_{a} B^{\mathcal{J}}=i\left(\gamma^{5}\right)_{a}{ }^{b} \psi_{b}^{\mathcal{J}}, \\
\mathrm{D}_{a} F^{\mathcal{J}}= & \left(\gamma^{0}\right)_{a}{ }^{b} \psi_{b}^{\mathcal{J}}, \quad \mathrm{D}_{a} G^{\mathcal{J}}=i\left(\gamma^{5} \gamma^{0}\right)_{a}{ }^{b} \psi_{b}^{\mathcal{J}}, \\
\mathrm{D}_{a} \psi_{b}^{\mathcal{J}}= & i\left(\gamma^{0}\right)_{a b}\left(\partial_{\tau} A^{\mathcal{J}}\right)-\left(\gamma^{5} \gamma^{0}\right)_{a b}\left(\partial_{\tau} B^{\mathcal{J}}\right) \\
& -i C_{a b}\left(\partial_{\tau} F^{\mathcal{J}}\right)+\left(\gamma^{5}\right)_{a b}\left(\partial_{\tau} G^{\mathcal{J}}\right), \\
\mathrm{D}_{a} A_{m}= & \left(\gamma_{m}\right)_{a}{ }^{b} \lambda_{b}, \quad \mathrm{D}_{a} \mathrm{~d}=i\left(\gamma^{5} \gamma^{0}\right)_{a}{ }^{b} \lambda_{b}, \\
\mathrm{D}_{a} \lambda_{b}= & -i\left(\gamma^{0} \gamma^{m}\right)_{a b}\left(\partial_{\tau} A_{m}\right)+\left(\gamma^{5}\right)_{a b}\left(\partial_{\tau} \mathrm{d}\right), \\
\mathrm{D}_{a}^{\mathcal{I}} A^{\mathcal{J}}= & \delta^{\mathcal{I} \mathcal{J}} \lambda_{a}-\epsilon^{\mathcal{I} \mathcal{J}} \psi_{\mathcal{K}}^{\mathcal{K}}, \\
\mathrm{D}_{a}^{\mathcal{I}} B^{\mathcal{J}}= & i\left(\gamma^{5}\right)_{a}{ }^{b}\left[\delta^{I J} \lambda_{b}+\epsilon^{\mathcal{I} \mathcal{J}} \psi_{\mathcal{K}}^{\mathcal{K}}\right], \\
\mathrm{D}_{a}^{\mathcal{I}} F^{\mathcal{J}}= & \left(\gamma^{0}\right)_{a}{ }^{b}\left[\delta^{\mathcal{I} \mathcal{J}} \lambda_{b}-\epsilon^{\mathcal{I} \mathcal{J}} \psi_{\mathcal{K}} \psi_{b}^{\mathcal{K}}\right], \\
\mathrm{D}_{a}^{\mathcal{I}} G^{\mathcal{J}}= & i\left(\gamma^{5} \gamma^{0}\right)_{a}{ }^{b}\left[-\delta^{I J} \lambda_{b}+\epsilon^{\mathcal{I} \mathcal{J}} \psi_{\mathcal{K}}^{\mathcal{K}}\right], \\
\mathrm{D}_{a}^{\mathcal{I}} \psi_{b}^{\mathcal{J}}= & \delta^{\mathcal{I} \mathcal{J}}\left[i\left(\gamma^{0} \gamma^{m}\right)_{a b}\left(\partial_{\tau} A_{m}\right)+\left(\gamma^{5}\right)_{a b}\left(\partial_{\tau} \mathrm{d}\right)\right] \\
& +\epsilon^{\mathcal{I} \mathcal{J}}{ }_{\mathcal{K}}\left[i\left(\gamma^{0}\right)_{a b}\left(\partial_{\tau} A^{\mathcal{K}}\right)+\left(\gamma^{5} \gamma^{0}\right)_{a b}\left(\partial_{\tau} B^{\mathcal{K}}\right)\right. \\
& \left.\quad-i C_{a b}\left(\partial_{\tau} F^{\mathcal{K}}\right)-\left(\gamma^{5}\right)_{a b}\left(\partial_{\tau} G^{\mathcal{K}}\right)\right], \\
\mathrm{D}_{a}^{\mathcal{I}} A_{m}= & -\left(\gamma_{m}\right)_{a}{ }^{b} \psi_{b}^{\mathcal{I}}, \quad \mathrm{D}_{a}^{\mathcal{I}} \mathrm{d}=i\left(\gamma^{5} \gamma^{0}\right)_{a}{ }^{b} \psi_{b}^{\mathcal{I}}, \\
\mathrm{D}_{a}^{\mathcal{I}} \lambda_{b}= & i\left(\gamma^{0}\right)_{a b}\left(\partial_{\tau} A^{\mathcal{I}}\right)-\left(\gamma^{5} \gamma^{0}\right)_{a b}\left(\partial_{\tau} B^{\mathcal{I}}\right) \\
& -i C_{a b}\left(\partial_{\tau} F^{\mathcal{I}}\right)-\left(\gamma^{5}\right)_{a b}\left(\partial_{\tau} G^{\mathcal{I}}\right),
\end{aligned}
$$

The three supersymmetries generated by $\mathrm{D}_{a}^{\mathcal{I}}$ are on-shell. If they were off-shell, the $\mathcal{N}=$ 4 extended version of (4.4) would read as

$$
\begin{aligned}
\left\{\mathrm{D}_{a}, \mathrm{D}_{b}\right\} & =i 2\left(\gamma^{0}\right)_{a b} \partial_{\tau}, \quad\left\{\mathrm{D}_{a}, \mathrm{D}_{b}^{\mathcal{I}}\right\}=0, \\
\left\{\mathrm{D}_{a}^{\mathcal{I}}, \mathrm{D}_{b}^{\mathcal{J}}\right\} & =i 2 \delta^{\mathcal{I} \mathcal{J}}\left(\gamma^{0}\right)_{a b} \partial_{\tau} .
\end{aligned}
$$

The explicit forms of the coefficients in (4.3) appropriate for this theory can now be read off from the equations in (4.7) then via direct calculation, it is found [26] that only the first equation in (4.8) is satisfied by the field content in (4.5).

The strongest interpretation of the Siegel-Roček No-Go Theorem to this 1D valise formulation would involve claiming there exists no possible extension of the field content vectors in (4.5) such that the equations in (4.8) can be satisfied. To reach this result, however, requires an assumption about the form of additional terms that must be added to (4.6) as in the original discussion.

\section{Within the world of adinkra network valise supermultiplets}

During the course of our efforts since the work of [27, 28], we have produced evidence suggesting there exists a way to apply the old Macintosh Mantra of "Think Different" to the problem stated in the previous section. 


$$
\mathcal{R}=\# 1
$$

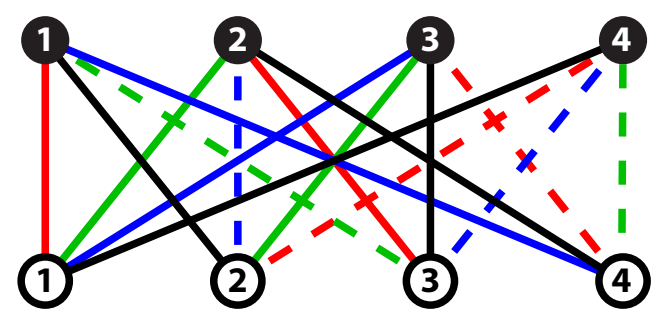

$$
\mathcal{R}=\# \mathbf{2}
$$

Figure 1. Two valise adinkra graphs with node assignment.

This alternative approach starts from networks that precisely encode the same kinematic information as the 0-brane-world description of valise supermultiplets. The graphical representations of these networks were given the name of "adinkras" [10]. Two examples of these are shown below.

We were led to these graphs by first discovering the adjacency matrices [27, 28] associated with them. These adjacency matrices satisfy a set of algebraic conditions we have named the $\mathcal{G} \mathcal{R}(\mathrm{d}, \mathcal{N})$ or "Garden Algebra" conditions and have been completely defined in the works of [11-14]. Via a set of Feynman-like rules (see e.g. [23]), these networks can be shown to be equivalent to equations

$$
\mathrm{D}_{\mathrm{I}} \Phi_{i}=i\left(\mathrm{~L}_{\mathrm{I}}\right)_{i \hat{k}} \Psi_{\hat{k}}, \quad \mathrm{D}_{\mathrm{I}} \Psi_{\hat{k}}=\left(\mathrm{R}_{\mathrm{I}}\right)_{\hat{k} i} \partial_{\tau} \Phi_{i} \Longrightarrow\left\{\mathrm{D}_{\mathrm{I}}, \mathrm{D}_{\mathrm{J}}\right\}=i 2 \delta_{\mathrm{IJ}} \partial_{\tau} .
$$

\subsection{Chiral supermultiplet adinkra network valise off-shell}

In the case of the first adinkra network shown in (figure 1), the L-matrices and R-matrices take the forms given by

$$
\begin{aligned}
& \left(\mathrm{L}_{1}\right)_{i \hat{k}}=\left[\begin{array}{cccc}
1 & 0 & 0 & 0 \\
0 & 0 & 0 & -1 \\
0 & 1 & 0 & 0 \\
0 & 0 & -1 & 0
\end{array}\right] \\
& \left(\mathrm{L}_{2}\right)_{i \hat{k}}=\left[\begin{array}{cccr}
0 & 1 & 0 & 0 \\
0 & 0 & 1 & 0 \\
-1 & 0 & 0 & 0 \\
0 & 0 & 0 & -1
\end{array}\right] \\
& \left(\mathrm{L}_{3}\right)_{i \hat{k}}=\left[\begin{array}{cccr}
0 & 0 & 1 & 0 \\
0 & -1 & 0 & 0 \\
0 & 0 & 0 & -1 \\
1 & 0 & 0 & 0
\end{array}\right] \text {, } \\
& \left(\mathrm{L}_{4}\right)_{i \hat{k}}=\left[\begin{array}{cccc}
0 & 0 & 0 & 1 \\
1 & 0 & 0 & 0 \\
0 & 0 & 1 & 0 \\
0 & 1 & 0 & 0
\end{array}\right] \text {. } \\
& \left(\mathrm{R}_{1}\right)_{\hat{k} i}=\left[\begin{array}{cccc}
1 & 0 & 0 & 0 \\
0 & 0 & 1 & 0 \\
0 & 0 & 0 & -1 \\
0 & -1 & 0 & 0
\end{array}\right] \text {, } \\
& \left(\mathrm{R}_{2}\right)_{\hat{k} i}=\left[\begin{array}{rrrr}
0 & 0 & -1 & 0 \\
1 & 0 & 0 & 0 \\
0 & 1 & 0 & 0 \\
0 & 0 & 0 & -1
\end{array}\right] \text {, } \\
& \left(\mathrm{R}_{3}\right)_{\hat{k} i}=\left[\begin{array}{rrrr}
0 & 0 & 0 & 1 \\
0 & -1 & 0 & 0 \\
1 & 0 & 0 & 0 \\
0 & 0 & -1 & 0
\end{array}\right] \text {, } \\
& \left(\mathrm{R}_{4}\right)_{\hat{k} i}=\left[\begin{array}{cccc}
0 & 1 & 0 & 0 \\
0 & 0 & 0 & 1 \\
0 & 0 & 1 & 0 \\
1 & 0 & 0 & 0
\end{array}\right] \text {. }
\end{aligned}
$$


These satisfy the Garden Algebra relationships

$$
\begin{aligned}
& \left(\mathrm{L}_{\mathrm{I}}\right)_{i}{ }^{\hat{\jmath}}\left(\mathrm{R}_{\mathrm{J}}\right)_{\hat{\jmath}}{ }^{k}+\left(\mathrm{L}_{\mathrm{J}}\right)_{i}{ }^{\hat{\jmath}}\left(\mathrm{R}_{\mathrm{I}}\right)_{\hat{\jmath}}{ }^{k}=2 \delta_{\mathrm{IJ}} \delta_{i}{ }^{k} \\
& \left(\mathrm{R}_{\mathrm{J}}\right)_{\hat{\imath}}{ }^{j}\left(\mathrm{~L}_{\mathrm{I}}\right)_{j}{ }^{\hat{k}}+\left(\mathrm{R}_{\mathrm{I}}\right)_{\hat{\imath}}{ }^{j}\left(\mathrm{~L}_{\mathrm{J}}\right)_{j}{ }^{\hat{k}}=2 \delta_{\mathrm{IJ}} \delta_{\hat{\imath}}^{\hat{k}}
\end{aligned}
$$

\subsection{Chiral supermultiplet adinkra network valise on-shell}

If we delete the open nodes denoted by 3 and 4 in the first adinkra labelled as $\mathcal{R}=\# 1$ as well as eliminate all the links associated with those nodes we find,

$$
\begin{array}{rlrl}
\left(\mathrm{L}_{1}\right)_{i \hat{k}}= & {\left[\begin{array}{cccc}
1 & 0 & 0 & 0 \\
0 & 0 & 0 & -1
\end{array}\right],} & \left(\mathrm{L}_{2}\right)_{i \hat{k}}=\left[\begin{array}{llll}
0 & 1 & 0 & 0 \\
0 & 0 & 1 & 0
\end{array}\right] \\
\left(\mathrm{L}_{3}\right)_{i \hat{k}}=\left[\begin{array}{cccc}
0 & 0 & 1 & 0 \\
0 & -1 & 0 & 0
\end{array}\right], & \left(\mathrm{L}_{4}\right)_{i \hat{k}}=\left[\begin{array}{llll}
0 & 0 & 0 & 1 \\
1 & 0 & 0 & 0
\end{array}\right] \\
\left(\mathrm{R}_{1}\right)_{\hat{k} i}=\left[\begin{array}{cc}
1 & 0 \\
0 & 0 \\
0 & 0 \\
0 & -1
\end{array}\right], & \left(\mathrm{R}_{2}\right)_{\hat{k} i}=\left[\begin{array}{ll}
0 & 0 \\
1 & 0 \\
0 & 1 \\
0 & 0
\end{array}\right] \\
\left(\mathrm{R}_{3}\right)_{\hat{k} i}=\left[\begin{array}{rr}
0 & 0 \\
0 & -1 \\
1 & 0 \\
0 & 0
\end{array}\right], & \left(\mathrm{R}_{4}\right)_{\hat{k} i}=\left[\begin{array}{ll}
0 & 1 \\
0 & 0 \\
0 & 0 \\
1 & 0
\end{array}\right]
\end{array}
$$

Given the matrices in (5.5) and (5.6) we find the following relations hold

$$
\begin{aligned}
& \left(\mathrm{L}_{\mathrm{I}}\right)_{i}^{\hat{\jmath}}\left(\mathrm{R}_{\mathrm{J}}\right)_{\hat{\jmath}}{ }^{k}+\left(\mathrm{L}_{\mathrm{J}}\right)_{i}^{\hat{\jmath}}\left(\mathrm{R}_{\mathrm{I}}\right)_{\hat{\jmath}}{ }^{k}=2 \delta_{\mathrm{IJ}} \delta_{i}{ }^{k} \\
& \left(\mathrm{R}_{\mathrm{J}}\right)_{\hat{\imath}}{ }^{j}\left(\mathrm{~L}_{\mathrm{I}}\right)_{j}{ }^{\hat{k}}+\left(\mathrm{R}_{\mathrm{I}}\right)_{\hat{\imath}}{ }^{j}\left(\mathrm{~L}_{\mathrm{J}}\right)_{j}{ }^{\hat{k}}=\delta_{\mathrm{IJ}} \delta_{\hat{\imath}}{ }^{\hat{k}}+\left[\vec{\alpha} \beta^{1}\right]_{\mathrm{IJ}} \cdot\left(\vec{\alpha} \beta^{1}\right)_{\hat{\imath}}{ }^{\hat{k}} .
\end{aligned}
$$

The six $4 \times 4$ matrices $\vec{\alpha}$ and $\vec{\beta}$ were defined in the work of [23].

It can be seen that the matrices of (5.2) and (5.3) are $\mathrm{d} \times \mathrm{d}$ matrices with $\mathrm{d}=4$. On the other hand the matrices in (5.5) are $\mathrm{d}_{L} \times \mathrm{d}_{R}$, and the matrices in (5.6) are $\mathrm{d}_{R} \times d_{L}$ where $\mathrm{d}_{L}=2$ and $\mathrm{d}_{R}=4$. We refer to matrices of this sort of structure as representatives of the $\mathcal{G R}\left(\mathrm{d}_{\mathbf{L}}, \mathrm{d}_{\mathbf{R}}, \mathcal{N}\right)$ algebra. 


\subsection{Vector supermultiplet adinkra network valise off-shell}

In the case of the second adinkra network shown in (figure 1), the L-matrices and R-matrices take the forms

$$
\begin{aligned}
& \left(\mathrm{L}_{1}\right)_{i \hat{k}}=\left[\begin{array}{cccc}
0 & 1 & 0 & 0 \\
0 & 0 & 0 & -1 \\
1 & 0 & 0 & 0 \\
0 & 0 & -1 & 0
\end{array}\right], \quad\left(\mathrm{L}_{2}\right)_{i \hat{k}}=\left[\begin{array}{cccc}
1 & 0 & 0 & 0 \\
0 & 0 & 1 & 0 \\
0 & -1 & 0 & 0 \\
0 & 0 & 0 & -1
\end{array}\right], \\
& \left(\mathrm{L}_{3}\right)_{i \hat{k}}=\left[\begin{array}{cccc}
0 & 0 & 0 & 1 \\
0 & 1 & 0 & 0 \\
0 & 0 & 1 & 0 \\
1 & 0 & 0 & 0
\end{array}\right], \quad\left(\mathrm{L}_{4}\right)_{i \hat{k}}=\left[\begin{array}{cccc}
0 & 0 & 1 & 0 \\
-1 & 0 & 0 & 0 \\
0 & 0 & 0 & -1 \\
0 & 1 & 0 & 0
\end{array}\right] \text {, } \\
& \left(\mathrm{R}_{1}\right)_{\hat{k} i}=\left[\begin{array}{cccc}
0 & 0 & 1 & 0 \\
1 & 0 & 0 & 0 \\
0 & 0 & 0 & -1 \\
0 & -1 & 0 & 0
\end{array}\right], \quad\left(\mathrm{R}_{2}\right)_{\hat{k} i}=\left[\begin{array}{cccc}
1 & 0 & 0 & 0 \\
0 & 0 & -1 & 0 \\
0 & 1 & 0 & 0 \\
0 & 0 & 0 & -1
\end{array}\right] \text {, } \\
& \left(\mathrm{R}_{3}\right)_{\hat{k} i}=\left[\begin{array}{cccc}
0 & 0 & 0 & 1 \\
0 & 1 & 0 & 0 \\
0 & 0 & 1 & 0 \\
1 & 0 & 0 & 0
\end{array}\right], \quad\left(\mathrm{R}_{4}\right)_{\hat{k} i}=\left[\begin{array}{cccc}
0 & -1 & 0 & 0 \\
0 & 0 & 0 & 1 \\
1 & 0 & 0 & 0 \\
0 & 0 & -1 & 0
\end{array}\right] \text {. }
\end{aligned}
$$

These also satisfy the relationships

$$
\begin{aligned}
& \left(\mathrm{L}_{\mathrm{I}}\right)_{i} \hat{\jmath}\left(\mathrm{R}_{\mathrm{J}}\right)_{\hat{\jmath}}^{k}+\left(\mathrm{L}_{\mathrm{J}}\right)_{i}^{\hat{\jmath}}\left(\mathrm{R}_{\mathrm{I}}\right)_{\hat{\jmath}}^{k}=2 \delta_{\mathrm{IJ}} \delta_{i}^{k}, \\
& \left(\mathrm{R}_{\mathrm{J}}\right)_{\hat{\imath}}{ }^{j}\left(\mathrm{~L}_{\mathrm{I}}\right)_{j}{ }^{\hat{k}}+\left(\mathrm{R}_{\mathrm{I}}\right)_{\hat{\imath}}^{j}\left(\mathrm{~L}_{\mathrm{J}}\right)_{j}^{\hat{k}}=2 \delta_{\mathrm{IJ}} \delta_{\hat{\imath}}^{\hat{k}} .
\end{aligned}
$$

\subsection{Vector supermultiplet adinkra network valise on-shell}

If we erase the fourth open node and its associated links, the forms of the associated adjacency-like matrices become

$$
\begin{aligned}
& \left(\mathrm{L}_{1}\right)_{i \hat{k}}=\left[\begin{array}{cccc}
0 & 1 & 0 & 0 \\
0 & 0 & 0 & -1 \\
1 & 0 & 0 & 0
\end{array}\right], \quad\left(\mathrm{L}_{2}\right)_{i \hat{k}}=\left[\begin{array}{cccc}
1 & 0 & 0 & 0 \\
0 & 0 & 1 & 0 \\
0 & -1 & 0 & 0
\end{array}\right] \\
& \left(\mathrm{L}_{3}\right)_{i \hat{k}}=\left[\begin{array}{cccc}
0 & 0 & 0 & 1 \\
0 & 1 & 0 & 0 \\
0 & 0 & 1 & 0
\end{array}\right], \quad\left(\mathrm{L}_{4}\right)_{i \hat{k}}=\left[\begin{array}{cccc}
0 & 0 & 1 & 0 \\
-1 & 0 & 0 & 0 \\
0 & 0 & 0 & -1
\end{array}\right] \text {, } \\
& \left(\mathrm{R}_{1}\right)_{\hat{k} i}=\left[\begin{array}{ccc}
0 & 0 & 1 \\
1 & 0 & 0 \\
0 & 0 & 0 \\
0 & -1 & 0
\end{array}\right], \quad\left(\mathrm{R}_{2}\right)_{\hat{k} i}=\left[\begin{array}{ccc}
1 & 0 & 0 \\
0 & 0 & -1 \\
0 & 1 & 0 \\
0 & 0 & 0
\end{array}\right] \text {, } \\
& \left(\mathrm{R}_{3}\right)_{\hat{k} i}=\left[\begin{array}{ccc}
0 & 0 & 0 \\
0 & 1 & 0 \\
0 & 0 & 1 \\
1 & 0 & 0
\end{array}\right], \quad\left(\mathrm{R}_{4}\right)_{\hat{k} i}=\left[\begin{array}{ccc}
0 & -1 & 1 \\
0 & 0 & 0 \\
1 & 0 & 0 \\
0 & 0 & -1
\end{array}\right] \text {, }
\end{aligned}
$$


The matrices in (5.8) and (5.9) lead to the following relations

$$
\begin{aligned}
&\left(\mathrm{L}_{\mathrm{I}}\right)_{i}^{\hat{\jmath}}\left(\mathrm{R}_{\mathrm{J}}\right)_{\hat{\jmath}}^{k}+\left(\mathrm{L}_{\mathrm{J}}\right)_{i}^{\hat{\jmath}}\left(\mathrm{R}_{\mathrm{I}}\right)_{\hat{\jmath}}^{k}=2 \delta_{\mathrm{IJ}} \delta_{i}^{k} \\
&\left(\mathrm{R}_{\mathrm{J}}\right)_{\hat{\imath}}{ }^{j}\left(\mathrm{~L}_{\mathrm{I}}\right)_{j}^{\hat{k}}+\left(\mathrm{R}_{\mathrm{I}}\right)_{\hat{\imath}}^{j}\left(\mathrm{~L}_{\mathrm{J}}\right)_{j}^{\hat{k}}= \frac{3}{2} \delta_{\mathrm{IJ}}\left(\mathrm{I}_{4}\right)_{\hat{\imath}}^{\hat{k}}-\frac{1}{2}\left[\vec{\alpha} \beta^{2}\right]_{\mathrm{IJ}} \cdot\left(\vec{\alpha} \beta^{2}\right)_{\hat{\imath}}^{\hat{k}} \\
&+\frac{1}{2}\left[\vec{\alpha} \beta^{1}\right]_{\mathrm{IJ}} \cdot\left(\vec{\alpha} \beta^{1}\right)_{\hat{\imath}}^{\hat{k}} \\
&+\frac{1}{2}\left[\vec{\alpha} \beta^{3}\right]_{\mathrm{IJ}} \cdot\left(\vec{\alpha} \beta^{3}\right)_{\hat{\imath}}^{\hat{k}} .
\end{aligned}
$$

At this stage, it is obvious that there are some interesting correlations between the calculations done from the $\gamma$-matrices of a 0 -brane-world starting point and similar calculations done from the basis of the adjacency matrices of an adinkra network-world starting point.

For the adinkra network valise chiral supermultiplet with adjacency matrices described by (5.2), and (5.3) the commutator algebra shown in (5.1) is satisfied on $\Phi_{i}$ and on $\Psi_{\hat{k}}$. For the adinkra network valise chiral supermultiplet with adjacency matrices described by (5.5) and (5.5) the commutator algebra shown in (5.1) is satisfied on $\Phi_{i}$, but not on $\Psi_{\hat{k}}$ due to the second line of (5.7).

For the adinkra network valise vector supermultiplet with adjacency matrices described by (5.8) and (5.9), the commutator algebra shown in (5.1) is satisfied on $\Phi_{i}$ and on $\Psi_{\hat{k}}$. For the adinkra network valise chiral supermultiplet with adjacency matrices described by (5.11) and (5.12) the commutator algebra shown in (5.1) is satisfied on $\Phi_{i}$, but not on $\Psi_{\hat{k}}$ due to the second line of (5.13).

\section{An adinkra network-world formulation of the off-shell SUSY auxiliary field problem}

The off-shell problem in the world of 0-brane valise supermultiplets can be recast into an equivalent one involving adinkra valise networks. There is one important difference however. As the starting point is in terms of adinkra networks, there is no information a priori about Lorentz representations.

Let $\Phi_{i}(\tau)$, and $\Psi_{\hat{i}}(\tau)$ denote arbitrary bosonic and fermionic sets of functions associated with the nodes of a valise adinkra. All the bosonic functions satisfy the equation

$$
\Phi_{i}\left(\tau_{1}\right) \Phi_{j}\left(\tau_{2}\right)=+\Phi_{j}\left(\tau_{2}\right) \Phi_{i}\left(\tau_{1}\right)
$$

and all the fermionic functions satisfy the equation

$$
\Psi_{\hat{i}}\left(\tau_{1}\right) \Psi_{\hat{k}}\left(\tau_{2}\right)=-\Psi_{\hat{k}}\left(\tau_{2}\right) \Psi_{\hat{i}}\left(\tau_{1}\right)
$$

The off-shell auxiliary field problem then asks that one determine all sets of bosonic functions $\Phi_{i}(\tau)$, sets of fermionic functions $\Psi_{\widehat{k}}(\tau)$, and associated matrices $\left(\mathrm{L}_{\mathrm{I}}\right)_{i \hat{k}}$ and $\left(\mathrm{R}_{\mathrm{I}}\right)_{\hat{k} i}$ to be used in the equations

$$
\mathrm{D}_{\mathrm{I}} \Phi_{i}=i\left(\mathrm{~L}_{\mathrm{I}}\right)_{i \hat{k}} \Psi_{\hat{k}}, \quad \mathrm{D}_{\mathrm{I}} \Psi_{\hat{k}}=\left(\mathrm{R}_{\mathrm{I}}\right)_{\hat{k} i} \partial_{\tau} \Phi_{i}
$$


Since the definitions of these L-matrices and R-matrices rely on the adinkras networks we have

$$
\begin{aligned}
& \left(\mathrm{L}_{\mathrm{I}}\right)_{i}^{\hat{\jmath}}\left(\mathrm{R}_{\mathrm{J}}\right)_{\hat{\jmath}}{ }^{k}+\left(\mathrm{L}_{\mathrm{J}}\right)_{i}^{\hat{\jmath}}\left(\mathrm{R}_{\mathrm{I}}\right)_{\hat{\jmath}}^{k}=2 \delta_{\mathrm{IJ}} \delta_{i}^{k}, \\
& \left(\mathrm{R}_{\mathrm{J}}\right)_{\hat{\imath}}{ }^{j}\left(\mathrm{~L}_{\mathrm{I}}\right)_{j}^{\hat{k}}+\left(\mathrm{R}_{\mathrm{I}}\right)_{\hat{\imath}}^{j}\left(\mathrm{~L}_{\mathrm{J}}\right)_{j}^{\hat{k}}=2 \delta_{\mathrm{IJ}} \delta_{\hat{\imath}}^{\hat{k}},
\end{aligned}
$$

which then imply the result

$$
\left\{\mathrm{D}_{\mathrm{I}}, \mathrm{D}_{\mathrm{J}}\right\}=i 2 \delta_{\mathrm{IJ}} \partial_{\tau}
$$

on both bosonic and fermionic field variables. According to our previous studies of adinkras, this is a solved problem.

Thus the question becomes, "How can this information be used to address the offshell problem in adinkra network world?" Stated another way, if one is solely given the information in (5.5) and (5.6) how does one recover (5.2) and (5.3) for the chiral supermultiplet adinkra? Alternately, given solely the information in (5.11) and (5.12) how does one recover (5.8) and (5.9) for the vector supermultiplet adinkra?

This will be addressed in the next section with the introduction of the concept of "On-Shell Adinkra-Network Deformations."

\section{On-shell adinkra network deformations}

When one reviews the arguments and equations of section four in comparison with those in section six, it may seems as though the problems are the same.

The 0-brane-world formulation begins with bosonic variables $\Phi_{\Lambda}$ and fermionic variables $\Psi_{\widehat{\Lambda}}$ in equations of the form

$$
\mathrm{D}_{a} \Phi_{\Lambda}=i\left(\mathrm{~L}_{\Lambda}\right)_{a}{ }^{\widehat{\Lambda}} \Psi_{\widehat{\Lambda}}, \quad \mathrm{D}_{a} \Psi_{\widehat{\Lambda}}=\left(\mathrm{R}^{\Lambda}\right)_{\widehat{\Lambda} a} \partial_{\tau} \Phi_{\Lambda},
$$

that ought then necessarily imply

$$
\left\{\mathrm{D}_{a}, \mathrm{D}_{b}\right\}=i 2\left(\gamma^{0}\right)_{a b} \partial_{\tau},
$$

to describe an off-shell supermultiplet.

The adinkra-network world formulation begins with bosonic variables $\Phi_{i}$ and fermionic variables $\Psi_{\hat{k}}$ in equations of the form

$$
\mathrm{D}_{\mathrm{I}} \Phi_{i}=i\left(\mathrm{~L}_{\mathrm{I}}\right)_{i \hat{k}} \Psi_{\hat{k}}, \quad \mathrm{D}_{\mathrm{I}} \Psi_{\hat{k}}=\left(\mathrm{R}_{\mathrm{I}}\right)_{\hat{k} i} \partial_{\tau} \Phi_{i}
$$

that ought then necessarily imply

$$
\left\{\mathrm{D}_{\mathrm{I}}, \mathrm{D}_{\mathrm{J}}\right\}=i 2 \delta_{\mathrm{IJ}} \partial_{\tau}
$$

The similarities between (7.1) and (7.2) on the one hand and (7.3) and (7.4) on the other are striking. However, computationally and operationally there are subtle differences.

In order to go from (7.1) to (7.2) one must

(1a.) make ansatzë for the coefficients $\left(\mathrm{L}_{\Lambda}\right)$ and $\left(\mathrm{R}^{\Lambda}\right)$, 
(1b.) make ansatzë for the field content vectors $\Phi_{\Lambda}$ and $\Psi_{\widehat{\Lambda}}$,

(1c.) calculate a set of matrix equations involving $\left(\mathrm{L}_{\Lambda}\right)$ and $\left(\mathrm{R}^{\Lambda}\right)$ to evaluate on all the bosonic fields, and

(1d.) calculate a set of Fierz identities involving $\left(\mathrm{L}_{\Lambda}\right)$ and $\left(\mathrm{R}^{\Lambda}\right)$ to evaluate on all the fermionic fields.

This last step is so because the quantities $\left(\mathrm{L}_{\Lambda}\right)$ and $\left(\mathrm{R}^{\Lambda}\right)$ are constructed from $\gamma$-matrices and the evaluation of (7.2) acting on fermions in a valise supermultiplet requires evaluation of Fierz Identities.

In order to go from (7.3) to (7.4) one must

(2a. make ansatzë for the coefficients $\left(\mathrm{L}_{\mathrm{I}}\right)$ and $\left(\mathrm{R}_{\mathrm{I}}\right)$,

(2b.) make ansatzë for the field content vectors $\Phi_{i}$ and $\Psi_{\hat{k}}$,

(2c.) calculate a set of matrix equations involving $\left(\mathrm{L}_{\mathrm{I}}\right)$ and $\left(\mathrm{R}_{\mathrm{I}}\right)$ to evaluate on all the bosonic fields, and

(2d.) calculate a set of matrix equations involving $\left(\mathrm{L}_{\mathrm{I}}\right)$ and $\left(\mathrm{R}_{\mathrm{I}}\right)$ to evaluate on all the fermionic fields.

Obtaining (7.4) from (7.3) does not require knowledge of Fierz Identities as the quantities $\left(\mathrm{L}_{\mathrm{I}}\right)$ and $\left(\mathrm{R}_{\mathrm{I}}\right)$ are constructed from adinkra network related matrices and only matrix multiplication is required to evaluate (7.4) on both bosons and fermions. This latter distinction makes for a substantial difference in the design of algorithms to search for possible auxiliary fields.

Another savings in required computation occurs because of differences in field content vectors required for their respective ansatë. In the case of $\Phi_{\Lambda}$ and $\Psi_{\widehat{\Lambda}}$ one must include data about the space-time spin of the component fields. In the case of $\Phi_{i}$ and $\Psi_{\hat{k}}$ all one has to do is to require that the range of their respective indices goes from 1 to multiples of 4 . As the spin bundle information of the fields is considerable, any calculation involving them must keep track of this information.

One of the results of our previous work is it appears such spin-bundle information emerges from the adinkra networks. In other words, by working with component fields that only depend on time and possess an $\mathrm{SU}(2) \times \mathrm{SU}(2)$ symmetry, embedded within four color networks seems to allow the isospin of the network to completely carry the spin bundle information for free.

We consider how to create algorithms to go from an on-shell adinkra network to an off-shell one.

As we have seen (see (5.5) and (5.6) for the on-shell chiral adinkra network and (5.8) and (5.9) for the on-shell vector adinkra network), in on-shell adinkra networks only some of the rows or columns are given due to the on-shell nature of the representation. So the unknown entries in the L-matrices and R-matrices can be represented by real parameters we will denote by the symbol $\ell$. These may be used to augment the rows and columns of 
the L-matrices and R-matrices until one reaches a $4 p \times 4 p$ matrix in all cases for some integer $p$. This is explicitly shown in equations (7.9), (7.10), (7.24), and (7.25) below. The problem of going from the on-shell adinkra network to a corresponding off-shell one, has now been reduced to the problem of determining the values of the $\ell$-parameters in the augmented matrices so as to satisfy the conditions in (5.4).

It is amusing to note that this problem is roughly analogous to a cryptographic one. The on-shell forms of the L-matrices and R-matrices all together for any particular valise supermultiplet play the role of an encrypted message and finding the corresponding off-shell L-matrices and R-matrices is analogous to decoding the message.

We now need to specify a series of operations to achieve this. The key to achieving this is the Garden Algebra (5.4). These conditions can be separated into four different parts

$$
\begin{aligned}
\left(\mathrm{L}_{\mathrm{I}}\right)_{i}^{\hat{\jmath}}\left(\mathrm{R}_{\mathrm{J}}\right)_{\hat{\jmath}}^{k}+\left(\mathrm{L}_{\mathrm{J}}\right)_{i}^{\hat{\jmath}}\left(\mathrm{R}_{\mathrm{I}}\right)_{\hat{\jmath}}^{k}=0 \text { where } I \neq J . \\
\left(\mathrm{R}_{\mathrm{J}}\right)_{\hat{\imath}}{ }^{j}\left(\mathrm{~L}_{\mathrm{I}}\right)_{j}^{\hat{k}}+\left(\mathrm{R}_{\mathrm{I}}\right)_{\hat{\imath}}{ }^{j}\left(\mathrm{~L}_{\mathrm{J}}\right)_{j}{ }^{\hat{k}}=0 \text { where } I \neq J . \\
\left(\mathrm{L}_{\mathrm{I}}\right)_{i}^{\hat{\jmath}}\left(\mathrm{R}_{\mathrm{J}}\right)_{\hat{\jmath}}{ }^{k}=\delta_{i}{ }^{k} \quad \text { where } I=J . \\
\left(\mathrm{R}_{\mathrm{J}}\right)_{\hat{\imath}}{ }^{j}\left(\mathrm{~L}_{\mathrm{I}}\right)_{j}{ }^{\hat{k}}=\delta_{\hat{\imath}}^{\hat{k}} \text { where } I=J .
\end{aligned}
$$

In the subsequent discussion, we show how the use of these for the augmented on-shell L-matrices and R-matrices leads from on-shell results to off-shell ones in the case of the chiral and vector adinkra networks.

\subsection{On-shell chiral valise matrix deformation}

Define four matrices $\mathrm{L}_{\mathrm{I}}$ where $\mathrm{I}=1,2,3$, or 4 that also depend on eight continuous real variables denoted by $\ell_{31}, \ell_{32}, \ell_{33}, \ell_{34}, \ell_{41}, \ell_{42}, \ell_{43}$, and $\ell_{44}$, via the four equations

$$
\begin{aligned}
\left(\mathrm{L}_{1}\right)_{i \hat{k}}= & {\left.\left[\begin{array}{cccc}
1 & 0 & 0 & 0 \\
0 & 0 & 0 & -1 \\
\ell_{31} & \ell_{32} & \ell_{33} & \ell_{34} \\
\ell_{41} & \ell_{42} & \ell_{43} & \ell_{44}
\end{array}\right], \mathrm{L}_{2}\right)_{i \hat{k}}=\left[\begin{array}{cccc}
0 & 1 & 0 & 0 \\
0 & 0 & 1 & 0 \\
-\ell_{32} & \ell_{31} & -\ell_{34} & \ell_{33} \\
-\ell_{42} & \ell_{41} & -\ell_{44} & \ell_{43}
\end{array}\right], } \\
\left(\mathrm{L}_{3}\right)_{i \hat{k}}= & {\left[\begin{array}{cccc}
0 & 0 & 1 & 0 \\
0 & -1 & 0 & 0 \\
-\ell_{33} & \ell_{34} & \ell_{31} & -\ell_{32} \\
-\ell_{43} & \ell_{44} & \ell_{41} & -\ell_{42}
\end{array}\right],\left(\mathrm{L}_{4}\right)_{i \hat{k}}=\left[\begin{array}{cccc}
0 & 0 & 0 & 1 \\
1 & 0 & 0 & 0 \\
-\ell_{34} & -\ell_{33} & \ell_{32} & \ell_{31} \\
-\ell_{44} & -\ell_{43} & \ell_{42} & \ell_{41}
\end{array}\right] . }
\end{aligned}
$$

We find that a corresponding set of R-matrices to satisfy (7.5) is given by

$$
\begin{aligned}
& \left(\mathrm{R}_{1}\right)_{\hat{k} i}=\left[\begin{array}{cccc}
1 & 0 & \ell_{31} & \ell_{41} \\
0 & 0 & \ell_{32} & \ell_{42} \\
0 & 0 & \ell_{33} & \ell_{43} \\
0 & -1 & \ell_{34} & \ell_{44}
\end{array}\right], \quad\left(\mathrm{R}_{2}\right)_{\hat{k} i}=\left[\begin{array}{cccc}
0 & 0 & -\ell_{32} & -\ell_{42} \\
1 & 0 & \ell_{31} & \ell_{41} \\
0 & 1 & -\ell_{34} & -\ell_{44} \\
0 & 0 & \ell_{33} & \ell_{43}
\end{array}\right] \text {, } \\
& \left(\mathrm{R}_{3}\right)_{\hat{k} i}=\left[\begin{array}{cccc}
0 & 0 & -\ell_{33} & -\ell_{43} \\
0 & -1 & \ell_{34} & \ell_{44} \\
1 & 0 & \ell_{31} & \ell_{41} \\
0 & 0 & -\ell_{32} & -\ell_{42}
\end{array}\right], \quad\left(\mathrm{R}_{4}\right)_{\hat{k} i}=\left[\begin{array}{cccc}
0 & 1 & -\ell_{34} & -\ell_{44} \\
0 & 0 & -\ell_{33} & -\ell_{43} \\
0 & 0 & \ell_{32} & \ell_{42} \\
1 & 0 & \ell_{31} & \ell_{41}
\end{array}\right] \text {. }
\end{aligned}
$$


However, we can use these augmented L-matrices and R-matrices to carry out the calculations indicated in (7.6). For these calculations we find

$$
\begin{aligned}
\left(\mathrm{R}_{1}\right)_{\hat{\imath}}{ }^{j}\left(\mathrm{~L}_{2}\right)_{j}{ }^{\hat{k}}+\left(\mathrm{R}_{2}\right)_{\hat{\imath}}{ }^{j}\left(\mathrm{~L}_{1}\right)_{j}{ }^{\hat{k}}= & \\
& {\left[\begin{array}{cccc}
-2 \mathcal{P}_{11}^{[1 \mid 2]} & 1+\mathcal{P}_{12}^{[1 \mid 2]} & -\mathcal{P}_{13}^{[1 \mid 2]} & \mathcal{P}_{14}^{[1 \mid 2]} \\
1+\mathcal{P}_{12}^{[1 \mid 2]} & 2 \mathcal{P}_{11}^{[1 \mid 2]} & \mathcal{P}_{14}^{[1 \mid 2]} & \mathcal{P}_{13}^{[1 \mid 2]} \\
-\mathcal{P}_{13}^{[1 \mid 2]} & \mathcal{P}_{14}^{[1 \mid 2]} & -2 \mathcal{P}_{33}^{[1 \mid 2]} & -1+\mathcal{P}_{34}^{[1 \mid 2]} \\
\mathcal{P}_{14}^{[1 \mid 2]} & \mathcal{P}_{13}^{[1 \mid 2]} & -1+\mathcal{P}_{34}^{[1 \mid 2]} & 2 \mathcal{P}_{33}^{[1 \mid 2]}
\end{array}\right] }
\end{aligned}
$$

where

$$
\begin{aligned}
& \mathcal{P}_{11}^{[1 \mid 2]}=\ell_{31} \ell_{32}+\ell_{41} \ell_{42}, \quad \mathcal{P}_{12}^{[1 \mid 2]}=\ell_{31}^{2}-\ell_{32}^{2}+\ell_{41}^{2}-\ell_{42}^{2}, \\
& \mathcal{P}_{13}^{[1 \mid 2]}=\ell_{32} \ell_{33}+\ell_{31} \ell_{34}+\ell_{42} \ell_{43}+\ell_{41} \ell_{44}, \\
& \mathcal{P}_{14}^{[1 \mid 2]}=\ell_{31} \ell_{33}-\ell_{32} \ell_{34}+\ell_{41} \ell_{43}-\ell_{42} \ell_{44}, \\
& \mathcal{P}_{33}^{[1 \mid 2]}=\ell_{33} \ell_{34}+\ell_{43} \ell_{44}, \quad \mathcal{P}_{34}^{[1 \mid 2]}=\ell_{33}^{2}-\ell_{34}^{2}+\ell_{43}^{2}-\ell_{44}^{2}, \\
& \left(\mathrm{R}_{1}\right)_{\hat{\imath}}{ }^{j}\left(\mathrm{~L}_{3}\right)_{j}{ }^{\hat{k}}+\left(\mathrm{R}_{3}\right)_{\hat{\imath}}{ }^{j}\left(\mathrm{~L}_{1}\right)_{j}^{\hat{k}}= \\
& {\left[\begin{array}{cccc}
-2 \mathcal{P}_{11}^{[1 \mid 3]} & -\mathcal{P}_{12}^{[1 \mid 3]} & 1+\mathcal{P}_{13}^{[1 \mid 3]} & -\mathcal{P}_{14}^{[1 \mid 3]} \\
-\mathcal{P}_{12}^{[1 \mid 3]} & 2 \mathcal{P}_{22}^{[1 \mid 3]} & \mathcal{P}_{14}^{[1 \mid 3]} & 1-\mathcal{P}_{24}^{[1 \mid 3]} \\
1+\mathcal{P}_{13}^{[1 \mid 3]} & \mathcal{P}_{14}^{[1 \mid 3]} & 2 \mathcal{P}_{11}^{[1 \mid 3]} & -\mathcal{P}_{12}^{[1 \mid 3]} \\
-\mathcal{P}_{14}^{[1 \mid 3]} & 1-\mathcal{P}_{24}^{[1 \mid 3]} & -\mathcal{P}_{12}^{[1 \mid 3]} & -2 \mathcal{P}_{22}^{[1 \mid 3]}
\end{array}\right],}
\end{aligned}
$$

where

$$
\begin{gathered}
\mathcal{P}_{11}^{[1 \mid 3]}=\ell_{31} \ell_{33}+\ell_{41} \ell_{43}, \quad \mathcal{P}_{13}^{[1 \mid 3]}=\ell_{31}^{2}-\ell_{33}^{2}+\ell_{41}^{2}-\ell_{43}^{2}, \\
\mathcal{P}_{12}^{[1 \mid 3]}=\ell_{32} \ell_{33}-\ell_{31} \ell_{34}+\ell_{42} \ell_{43}-\ell_{41} \ell_{44}, \\
\mathcal{P}_{14}^{[1 \mid 3]}=\ell_{31} \ell_{32}+\ell_{33} \ell_{34}+\ell_{41} \ell_{42}+\ell_{43} \ell_{44}, \\
\mathcal{P}_{22}^{[1 \mid 3]}=\ell_{32} \ell_{34}+\ell_{42} \ell_{44}, \quad \mathcal{P}_{24}^{[1 \mid 3]}=\ell_{32}^{2}-\ell_{34}^{2}+\ell_{42}^{2}-\ell_{44}^{2}, \\
\left(\mathrm{R}_{1}\right)_{\hat{\imath}}{ }^{j}\left(\mathrm{~L}_{4}\right)_{j}{ }^{\hat{k}}+\left(\mathrm{R}_{4}\right)_{\hat{\imath}}{ }^{j}\left(\mathrm{~L}_{1}\right)_{j}^{\hat{k}}= \\
{\left[\begin{array}{cccc}
-2 \mathcal{P}_{11}^{[1 \mid 4]} & -\mathcal{P}_{12}^{[1 \mid 4]} & \mathcal{P}_{13}^{[1 \mid 4]} & \mathcal{P}_{14}^{[1 \mid 4]} \\
-\mathcal{P}_{12}^{[1 \mid 4]} & -2 \mathcal{P}_{22}^{[1 \mid 4]} & \mathcal{P}_{23}^{[1 \mid 4]} & \mathcal{P}_{13}^{[1 \mid 4]} \\
\mathcal{P}_{13}^{[1 \mid 4]} & \mathcal{P}_{23}^{[1 \mid 4]} & 2 \mathcal{P}_{22}^{[1 \mid 4]} & \mathcal{P}_{12}^{[1 \mid 4]} \\
\mathcal{P}_{14}^{[1 \mid 4]} & \mathcal{P}_{13}^{[1 \mid 4]} & \mathcal{P}_{12}^{[1 \mid 4]} & 2 \mathcal{P}_{11}^{[1 \mid 4]}
\end{array}\right],}
\end{gathered}
$$

where

$$
\begin{aligned}
& \mathcal{P}_{11}^{[1 \mid 4]}=\ell_{31} \ell_{34}+\ell_{41} \ell_{44}, \mathcal{P}_{14}^{[1 \mid 4]}=\ell_{31}^{2}-\ell_{34}^{2}+\ell_{41}^{2}-\ell_{44}^{2}, \\
& \mathcal{P}_{12}^{[1 \mid 4]}=\ell_{31} \ell_{33}+\ell_{32} \ell_{34}+\ell_{41} \ell_{43}+\ell_{42} \ell_{44}, \\
& \mathcal{P}_{13}^{[1 \mid 4]}=\ell_{31} \ell_{32}-\ell_{33} \ell_{34}+\ell_{41} \ell_{42}-\ell_{43} \ell_{44}, \\
& \mathcal{P}_{22}^{[1 \mid 4]}=\ell_{32} \ell_{33}+\ell_{42} \ell_{43}, \mathcal{P}_{23}^{[1 \mid 4]}=\ell_{32}^{2}-\ell_{33}^{2}+\ell_{42}^{2}-\ell_{43}^{2},
\end{aligned}
$$




$$
\begin{aligned}
&\left(\mathrm{R}_{2}\right)_{\hat{\imath}}{ }^{j}\left(\mathrm{~L}_{3}\right)_{j} \hat{k}^{\hat{k}}+\left(\mathrm{R}_{3}\right)_{\hat{i}}{ }^{j}\left(\mathrm{~L}_{2}\right)^{\hat{k}}= \\
& {\left[\begin{array}{cccc}
2 \mathcal{P}_{11}^{[2 \mid 3]} & -\mathcal{P}_{12}^{[2 \mid 3]} & -\mathcal{P}_{13}^{[2 \mid 3]} & \mathcal{P}_{14}^{[2 \mid 3]} \\
-\mathcal{P}_{12}^{[2 \mid 3]} & 2 \mathcal{P}_{22}^{[2 \mid 3]} & \mathcal{P}_{23}^{[2 \mid 3]} & -\mathcal{P}_{13}^{[2 \mid 3]} \\
-\mathcal{P}_{13}^{[2 \mid 3]} & \mathcal{P}_{23}^{[2 \mid 3]} & -2 \mathcal{P}_{22}^{[2 \mid 3]} & \mathcal{P}_{12}^{[2 \mid 3]} \\
\mathcal{P}_{14}^{[2 \mid 3]} & -\mathcal{P}_{13}^{[2 \mid 3]} & \mathcal{P}_{12}^{[2 \mid 3]} & -2 \mathcal{P}_{11}^{[2 \mid 3]}
\end{array}\right], }
\end{aligned}
$$

where

$$
\begin{aligned}
& \mathcal{P}_{11}^{[2 \mid 3]}=\ell_{32} \ell_{33}+\ell_{42} \ell_{43}, \mathcal{P}_{14}^{[2 \mid 3]}=\ell_{32}^{2}-\ell_{33}^{2}+\ell_{42}^{2}-\ell_{43}^{2}, \\
& \mathcal{P}_{12}^{[2 \mid 3]}=\ell_{31} \ell_{33}+\ell_{32} \ell_{34}+\ell_{41} \ell_{43}+\ell_{42} \ell_{44}, \\
& \mathcal{P}_{13}^{[2 \mid 3]}=\ell_{31} \ell_{32}-\ell_{33} \ell_{34}+\ell_{41} \ell_{42}-\ell_{43} \ell_{44}, \\
& \mathcal{P}_{22}^{[2 \mid 3]}=\ell_{31} \ell_{34}+\ell_{41} \ell_{44}, \mathcal{P}_{23}^{[2 \mid 3]}=\ell_{31}^{2}-\ell_{34}^{2}+\ell_{41}^{2}-\ell_{44}^{2}, \\
& \left(\mathrm{R}_{2}\right)_{\hat{\imath}}^{j}\left(\mathrm{~L}_{4}\right)_{j}{ }^{\hat{k}}+\left(\mathrm{R}_{4}\right)_{\hat{\imath}}{ }^{j}\left(\mathrm{~L}_{2}\right)_{j}^{\hat{k}}= \\
& {\left[\begin{array}{cccc}
2 \mathcal{P}_{11}^{[2 \mid 4]} & \mathcal{P}_{12}^{[2 \mid 4]} & 1-\mathcal{P}_{13}^{[2 \mid 4]} & -\mathcal{P}_{14}^{[2 \mid 4]} \\
\mathcal{P}_{12}^{[2 \mid 4]} & -2 \mathcal{P}_{22}^{[2 \mid 4]} & \mathcal{P}_{14}^{[2 \mid 4]} & 1+\mathcal{P}_{24}^{[2 \mid 4]} \\
1-\mathcal{P}_{13}^{[2 \mid 4]} & \mathcal{P}_{14}^{[2 \mid 4]} & -2 \mathcal{P}_{11}^{[2 \mid 4]} & \mathcal{P}_{12}^{[2 \mid 4]} \\
-\mathcal{P}_{14}^{[2 \mid 4]} & 1+\mathcal{P}_{24}^{[2 \mid 4]} & \mathcal{P}_{12}^{[2 \mid 4]} & 2 \mathcal{P}_{22}^{[2 \mid 4]}
\end{array}\right],}
\end{aligned}
$$

where

$$
\begin{gathered}
\mathcal{P}_{11}^{[2 \mid 4]}=\ell_{32} \ell_{34}+\ell_{42} \ell_{44}, \mathcal{P}_{13}^{[2 \mid 4]}=\ell_{32}^{2}-\ell_{34}^{2}+\ell_{42}^{2}-\ell_{44}^{2}, \\
\mathcal{P}_{12}^{[2 \mid 4]}=\ell_{32} \ell_{33}-\ell_{31} \ell_{34}+\ell_{42} \ell_{43}-\ell_{41} \ell_{44}, \\
\mathcal{P}_{14}^{[2 \mid 4]}=\ell_{31} \ell_{32}+\ell_{33} \ell_{34}+\ell_{41} \ell_{42}+\ell_{43} \ell_{44}, \\
\mathcal{P}_{22}^{[2 \mid 4]}=\ell_{31} \ell_{33}+\ell_{41} \ell_{43}, \mathcal{P}_{24}^{[2 \mid 4]}=\ell_{31}^{2}-\ell_{33}^{2}+\ell_{41}^{2}-\ell_{43}^{2}, \\
\left(\mathrm{R}_{3}\right)_{\hat{\imath}}{ }^{j}\left(\mathrm{~L}_{4}\right)_{j}{ }^{\hat{k}}+\left(\mathrm{R}_{4}\right)_{\hat{\imath}}{ }^{j}\left(\mathrm{~L}_{3}\right)_{j}{ }^{\hat{k}}= \\
{\left[\begin{array}{cccc}
2 \mathcal{P}_{11}^{[3 \mid 4]} & -1+\mathcal{P}_{12}^{[3 \mid 4]} & -\mathcal{P}_{13}^{[3 \mid 4]} & -\mathcal{P}_{14}^{[3 \mid 4]} \\
-1+\mathcal{P}_{12}^{[3 \mid 4]} & -2 \mathcal{P}_{11}^{[3 \mid 4]} & -\mathcal{P}_{14}^{[3 \mid 4]} & \mathcal{P}_{13}^{[3 \mid 4]} \\
-\mathcal{P}_{13}^{[3 \mid 4]} & -\mathcal{P}_{14}^{[3 \mid 4]} & 2 \mathcal{P}_{33}^{[3 \mid 4]} & 1+\mathcal{P}_{34}^{[3 \mid 4]} \\
-\mathcal{P}_{14}^{[3 \mid 4]} & \mathcal{P}_{13}^{[3 \mid 4]} & 1+\mathcal{P}_{34}^{[3 \mid 4]} & -2 \mathcal{P}_{33}^{[3 \mid 4]}
\end{array}\right],}
\end{gathered}
$$

where

$$
\begin{aligned}
& \mathcal{P}_{11}^{[3 \mid 4]}=\ell_{33} \ell_{34}+\ell_{43} \ell_{44}, \mathcal{P}_{12}^{[3 \mid 4]}=\ell_{33}^{2}-\ell_{34}^{2}+\ell_{43}^{2}-\ell_{44}^{2}, \\
& \mathcal{P}_{13}^{[3 \mid 4]}=\ell_{32} \ell_{33}+\ell_{31} \ell_{34}+\ell_{42} \ell_{43}+\ell_{41} \ell_{44}, \\
& \mathcal{P}_{14}^{[3 \mid 4]}=\ell_{31} \ell_{33}-\ell_{32} \ell_{34}+\ell_{41} \ell_{43}-\ell_{42} \ell_{44}, \\
& \mathcal{P}_{33}^{[3 \mid 4]}=\ell_{31} \ell_{32}+\ell_{41} \ell_{42}, \mathcal{P}_{34}^{[3 \mid 4]}=\ell_{31}^{2}-\ell_{32}^{2}+\ell_{41}^{2}-\ell_{42}^{2} .
\end{aligned}
$$

Imposing the conditions that the matrices in (7.11), (7.13), (7.15), (7.17), (7.19), and (7.21) should vanish yields solutions to these equations

$$
\ell_{32}= \pm 1, \quad \ell_{43}= \pm 1,
$$


and all other $\ell$-parameters vanish. Up to the field redefinitions $F \rightarrow-F$ and $G \rightarrow-G$, we have recovered the off-shell chiral adinkra network L-matrices and R-matrices by starting from the on-shell chiral adinkra network L-matrices and R-matrices. The solution in (7.23) also can be shown to satisfy the conditions in (7.7) and (7.8).

\subsection{On-shell vector valise matrix deformation}

Now we repeat the analysis of the previous subsection but switching our attention to the vector supermultiplet adinkra valise matrices. We introduce a set of deformation to the onshell L-matrices and R-matrices shown in (5.11) and (5.12) by introducing the deforming parameters $\ell_{41}, \ell_{42}, \ell_{43}$, and $\ell_{44}$ to augment the on-shell matrices according to

$$
\begin{aligned}
& \left(\mathrm{L}_{1}\right)_{i \hat{k}}=\left[\begin{array}{cccc}
0 & 1 & 0 & 0 \\
0 & 0 & 0 & -1 \\
1 & 0 & 0 & 0 \\
\ell_{41} & \ell_{42} & \ell_{43} & \ell_{44}
\end{array}\right], \quad\left(\mathrm{L}_{2}\right)_{i \hat{k}}=\left[\begin{array}{cccc}
1 & 0 & 0 & 0 \\
0 & 0 & 1 & 0 \\
0 & -1 & 0 & 0 \\
\ell_{42} & -\ell_{41} & -\ell_{44} & \ell_{43}
\end{array}\right], \\
& \left(\mathrm{L}_{3}\right)_{i \hat{k}}=\left[\begin{array}{cccc}
0 & 0 & 0 & 1 \\
0 & 1 & 0 & 0 \\
0 & 0 & 1 & 0 \\
-\ell_{43} & -\ell_{44} & \ell_{41} & \ell_{42}
\end{array}\right], \quad\left(\mathrm{L}_{4}\right)_{i \hat{k}}=\left[\begin{array}{cccc}
0 & 0 & 1 & 0 \\
-1 & 0 & 0 & 0 \\
0 & 0 & 0 & -1 \\
\ell_{44} & -\ell_{43} & \ell_{42} & -\ell_{41}
\end{array}\right], \\
& \left(\mathrm{R}_{1}\right)_{\hat{k} i}=\left[\begin{array}{cccc}
0 & 0 & 1 & \ell_{41} \\
1 & 0 & 0 & \ell_{42} \\
0 & 0 & 0 & \ell_{43} \\
0 & -1 & 0 & \ell_{44}
\end{array}\right], \quad\left(\mathrm{R}_{2}\right)_{\hat{k} i}=\left[\begin{array}{cccc}
1 & 0 & 0 & \ell_{42} \\
0 & 0 & -1 & -\ell_{41} \\
0 & 1 & 0 & -\ell_{44} \\
0 & 0 & 0 & \ell_{43}
\end{array}\right] \text {, } \\
& \left(\mathrm{R}_{3}\right)_{\hat{k} i}=\left[\begin{array}{cccc}
0 & 0 & 0 & -\ell_{43} \\
0 & 1 & 0 & -\ell_{44} \\
0 & 0 & 1 & \ell_{41} \\
1 & 0 & 0 & \ell_{42}
\end{array}\right], \quad\left(\mathrm{R}_{4}\right)_{\hat{k} i}=\left[\begin{array}{cccc}
0 & -1 & 0 & \ell_{44} \\
0 & 0 & 0 & -\ell_{43} \\
1 & 0 & 0 & \ell_{42} \\
0 & 0 & -1 & -\ell_{41}
\end{array}\right] \text {. }
\end{aligned}
$$

Once more direct calculations show these satisfy (7.5). However, we can also carry out similar calculations where the R-matrices appearing as the terms farthest to the left in the matrix multiplications. For these calculations we find

$$
\begin{aligned}
&\left(\mathrm{R}_{1}\right)_{\hat{\imath}}^{j}\left(\mathrm{~L}_{2}\right)_{j}^{\hat{k}}+\left(\mathrm{R}_{2}\right)_{\hat{\imath}}^{j}\left(\mathrm{~L}_{1}\right)_{j}^{\hat{k}}=\left.\begin{array}{cccc}
2 \ell_{41} \ell_{42} & -\ell_{41}^{2}+\ell_{42}^{2} & \ell_{42} \ell_{43}-\ell_{41} \ell_{44} & \ell_{41} \ell_{43}+\ell_{42} \ell_{44} \\
-\ell_{41}^{2}+\ell_{42}^{2} & -2 \ell_{41} \ell_{42} & -\ell_{41} \ell_{43}-\ell_{42} \ell_{44} & \ell_{42} \ell_{43}-\ell_{41} \ell_{44} \\
\ell_{42} \ell_{43}-\ell_{41} \ell_{44} & -\ell_{41} \ell_{43}-\ell_{42} \ell_{44} & -2 \ell_{43} \ell_{44} & -1+\ell_{43}^{2}-\ell_{44}^{2} \\
\ell_{41} \ell_{43}+\ell_{42} \ell_{44} & \ell_{42} \ell_{43}-\ell_{41} \ell_{44} & -1+\ell_{43}^{2}-\ell_{44}^{2} & 2 \ell_{43} \ell_{44}
\end{array}\right], \\
&\left(\mathrm{R}_{1}\right)_{\hat{\imath}}{ }^{j}\left(\mathrm{~L}_{3}\right)_{j}{ }^{\hat{k}}+\left(\mathrm{R}_{3}\right)_{\hat{\imath}}{ }^{j}\left(\mathrm{~L}_{1}\right)_{j}^{\hat{k}}= \\
& {\left[\begin{array}{cccc}
-2 \ell_{41} \ell_{43} & -\ell_{42} \ell_{43}-\ell_{41} \ell_{44} & 1+\ell_{41}^{2}-\ell_{43}^{2} & \ell_{41} \ell_{42}-\ell_{43} \ell_{44} \\
-\ell_{42} \ell_{43}-\ell_{41} \ell_{44} & -2 \ell_{42} \ell_{44} & \ell_{41} \ell_{42}-\ell_{43} \ell_{44} & \ell_{42}^{2}-\ell_{44}^{2} \\
1+\ell_{41}^{2}-\ell_{43}^{2} & \ell_{41} \ell_{42}-\ell_{43} \ell_{44} & 2 \ell_{41} \ell_{43} & \ell_{42} \ell_{43}+\ell_{41} \ell_{44} \\
\ell_{41} \ell_{42}-\ell_{43} \ell_{44} & \ell_{42}^{2}-\ell_{44}^{2} & \ell_{42} \ell_{43}+\ell_{41} \ell_{44} & 2 \ell_{42} \ell_{44}
\end{array}\right], }
\end{aligned}
$$




$$
\begin{aligned}
& \left(\mathrm{R}_{1}\right)_{\hat{\imath}}^{j}\left(\mathrm{~L}_{4}\right)_{j} \hat{k}+\left(\mathrm{R}_{4}\right)_{\hat{\imath}}{ }^{j}\left(\mathrm{~L}_{1}\right)_{j} \hat{k}= \\
& \quad\left[\begin{array}{cccc}
2 \ell_{41} \ell_{44} & -\ell_{41} \ell_{43}+\ell_{42} \ell_{44} & \ell_{41} \ell_{42}+\ell_{43} \ell_{44} & -\ell_{41}^{2}+\ell_{44}^{2} \\
-\ell_{41} \ell_{43}+\ell_{42} \ell_{44} & -2 \ell_{42} \ell_{43} & 1+\ell_{42}^{2}-\ell_{43}^{2} & -\ell_{41} \ell_{42}-\ell_{43} \ell_{44} \\
\ell_{41} \ell_{42}+\ell_{43} \ell_{44} & 1+\ell_{42}^{2}-\ell_{43}^{2} & 2 \ell_{42} \ell_{43} & -\ell_{41} \ell_{43}+\ell_{42} \ell_{44} \\
-\ell_{41}^{2}+\ell_{44}^{2} & -\ell_{41} \ell_{42}-\ell_{43} \ell_{44} & -\ell_{41} \ell_{43}+\ell_{42} \ell_{44} & -2 \ell_{41} \ell_{44}
\end{array}\right],
\end{aligned}
$$

$\left(\mathrm{R}_{2}\right)_{\hat{\imath}}^{j}\left(\mathrm{~L}_{3}\right)_{j}^{\hat{k}}+\left(\mathrm{R}_{3}\right)_{\hat{\imath}}^{j}\left(\mathrm{~L}_{2}\right)_{j}^{\hat{k}}=$

$$
\left[\begin{array}{cccc}
-2 \ell_{42} \ell_{43} & \ell_{41} \ell_{43}-\ell_{42} \ell_{44} & \ell_{41} \ell_{42}+\ell_{43} \ell_{44} & 1+\ell_{42}^{2}-\ell_{43}^{2} \\
\ell_{41} \ell_{43}-\ell_{42} \ell_{44} & 2 \ell_{41} \ell_{44} & -\ell_{41}^{2}+\ell_{44}^{2} & -\ell_{41} \ell_{42}-\ell_{43} \ell_{44} \\
\ell_{41} \ell_{42}+\ell_{43} \ell_{44} & -\ell_{41}^{2}+\ell_{44}^{2} & -2 \ell_{41} \ell_{44} & \ell_{41} \ell_{43}-\ell_{42} \ell_{44} \\
1+\ell_{42}^{2}-\ell_{43}^{2} & -\ell_{41} \ell_{42}-\ell_{43} \ell_{44} & \ell_{41} \ell_{43}-\ell_{42} \ell_{44} & 2 \ell_{42} \ell_{43}
\end{array}\right],
$$

$\left(\mathrm{R}_{2}\right)_{\hat{\imath}}^{j}\left(\mathrm{~L}_{4}\right)_{j}^{\hat{k}}+\left(\mathrm{R}_{4}\right)_{\hat{\imath}}^{j}\left(\mathrm{~L}_{2}\right)_{j}^{\hat{k}}=$

$$
\left[\begin{array}{cccc}
2 \ell_{42} \ell_{44} & -\ell_{42} \ell_{43}-\ell_{41} \ell_{44} & \ell_{42}^{2}-\ell_{44}^{2} & -\ell_{41} \ell_{42}+\ell_{43} \ell_{44} \\
-\ell_{42} \ell_{43}-\ell_{41} \ell_{44} & 2 \ell_{41} \ell_{43} & -\ell_{41} \ell_{42}+\ell_{43} \ell_{44} & 1+\ell_{41}^{2}-\ell_{43}^{2} \\
\ell_{42}^{2}-\ell_{44}^{2} & -\ell_{41} \ell_{42}+\ell_{43} \ell_{44} & -2 \ell_{42} \ell_{44} & \ell_{42} \ell_{43}+\ell_{41} \ell_{44} \\
-\ell_{41} \ell_{42}+\ell_{43} \ell_{44} & 1+\ell_{41}^{2}-\ell_{43}^{2} & \ell_{42} \ell_{43}+\ell_{41} \ell_{44} & -2 \ell_{41} \ell_{43}
\end{array}\right],
$$

$\left(\mathrm{R}_{3}\right)_{\hat{\imath}}^{j}\left(\mathrm{~L}_{4}\right)_{j}^{\hat{k}}+\left(\mathrm{R}_{4}\right)_{\hat{\imath}}^{j}\left(\mathrm{~L}_{3}\right)_{j}^{\hat{k}}=$

$$
\left[\begin{array}{cccc}
-2 \ell_{43} \ell_{44} & -1+\ell_{43}^{2}-\ell_{44}^{2} & -\ell_{42} \ell_{43}+\ell_{41} \ell_{44} & \ell_{41} \ell_{43}+\ell_{42} \ell_{44} \\
-1+\ell_{43}^{2}-\ell_{44}^{2} & 2 \ell_{43} \ell_{44} & -\ell_{41} \ell_{43}-\ell_{42} \ell_{44} & -\ell_{42} \ell_{43}+\ell_{41} \ell_{44} \\
-\ell_{42} \ell_{43}+\ell_{41} \ell_{44} & -\ell_{41} \ell_{43}-\ell_{42} \ell_{44} & 2 \ell_{41} \ell_{42} & -\ell_{41}^{2}+\ell_{42}^{2} \\
\ell_{41} \ell_{43}+\ell_{42} \ell_{44} & -\ell_{42} \ell_{43}+\ell_{41} \ell_{44} & -\ell_{41}^{2}+\ell_{42}^{2} & -2 \ell_{41} \ell_{42}
\end{array}\right] .
$$

If we impose the condition in (7.6) we are easily led to the solutions

$$
\ell_{41}=\ell_{42}=\ell_{44}=0, \quad \ell_{43}= \pm 1 .
$$

Up to a sign (which corresponds to the redefinition $d \rightarrow-d$ ) we recover the off-shell L-matrices and R-matrices of (5.8) and (5.9) for the adinkra network version of vector supermultiplet. The solution in (7.32) also can be shown to satisfy the conditions in (7.7) and (7.8).

So once again we see the method of deforming the on-shell matrices by augmentation involving the $\ell$-parameters followed by the imposition of the off-diagonal part of the Garden Algebra conditions leads from the on-shell to the off-shell versions of the matrices. To summarize the results of this section, we have shown that one can:

(a.) start with on-shell L-matrices and R-matrices (for the chiral adinkra network (5.5) and (5.6) or for the vector adinkra network (5.11) and (5.12)),

(b.) use $\ell$-parameters to augment the on-shell L-matrices and R-matrices (for the chiral adinkra network (7.9) and (7.10) or for the vector adinkra network (7.24) and (7.25)),

(c.) impose the Garden Algebra conditions in (7.5) and (7.6), and 
(d.) thereby, up to a set of field redefinitions, derive the off-shell versions of the respective L-matrices and R-matrices. (for the chiral adinkra network (5.2) and (5.3) or for the vector adinkra (5.8) and (5.9)).

The $\ell$-augmented L-matrices and R-matrices interpolate between the on-shell solutions (where all $\ell$ parameters vanish) and the off-shell ones (where the $\ell$ parameters take on the values shown in (7.23) or (7.32) in the respective cases). For general values of the $\ell$-parameters, the augmented matrices do not satisfy the Garden Algebra.

\section{The general cryptographic problem analogy to the adinkra network auxiliary field problem}

In this section, we want to discuss the general matrix problem that adinkra networks provide as the translation of the off-shell SUSY auxiliary field problem.

Consider a set of matrices of the forms

$$
\left(\mathrm{L}_{\mathrm{I}}\right)_{i \hat{k}}=\left[\begin{array}{cccccc}
a_{11}^{\mathrm{I}}+\ell_{11}^{\mathrm{I}} & a_{12}^{\mathrm{I}}+\ell_{12}^{\mathrm{I}} & \cdots & a_{1 r_{1}}^{\mathrm{I}}+\ell_{1 r_{1}}^{\mathrm{I}} & \cdots & a_{14 p}^{\mathrm{I}}+\ell_{14 p}^{\mathrm{I}} \\
a_{21}^{\mathrm{I}}+\ell_{21}^{\mathrm{I}} & a_{22}^{\mathrm{I}}+\ell_{22}^{\mathrm{I}} & \cdots & a_{2 r_{1}}^{\mathrm{I}}+\ell_{2 r_{1}}^{\mathrm{I}} & \cdots & a_{24 p}^{\mathrm{I}}+\ell_{24 p}^{\mathrm{I}} \\
\vdots & & & & & \vdots \\
\vdots & & & & & \vdots \\
\vdots & & & & & \vdots \\
\vdots & & & & & \vdots \\
a_{4 p 1}^{\mathrm{I}}+\ell_{4 p 1}^{\mathrm{I}} & a_{4 p 2}^{\mathrm{I}}+\ell_{4 p 2}^{\mathrm{I}} & \cdots & a_{4 p r_{1}}^{\mathrm{I}}+\ell_{4 p r_{1}}^{\mathrm{I}} & \cdots & a_{4 p 4 p}^{\mathrm{I}}+\ell_{4 p 4 p}^{\mathrm{I}}
\end{array}\right],
$$

and

$$
\left(\mathrm{R}_{\mathrm{I}}\right)_{\hat{k} i}=\left[\begin{array}{cccccc}
b_{11}^{\mathrm{I}}+\widehat{\ell}_{11}^{\mathrm{I}} & b_{12}^{\mathrm{I}}+\widehat{\ell}_{12}^{\mathrm{I}} & \cdots & b_{1 r_{1}}^{\mathrm{I}}+\widehat{\ell}_{1 r_{1}}^{\mathrm{I}} & \cdots & b_{14 p}^{\mathrm{I}}+\widehat{\ell}_{14 p}^{\mathrm{I}} \\
b_{21}^{\mathrm{I}}+\widehat{\ell}_{21}^{\mathrm{I}} & b_{22}^{\mathrm{I}}+\widehat{\ell}_{22}^{\mathrm{I}} & \cdots & b_{2 r_{1}}^{\mathrm{I}}+\widehat{\ell}_{2 r_{1}}^{\mathrm{I}} & \cdots & b_{24 p}^{\mathrm{I}}+\widehat{\ell}_{24 p}^{\mathrm{I}} \\
\vdots & & & & & \vdots \\
\vdots & & & & & \vdots \\
\vdots & & & & & \vdots \\
\vdots & & & & \vdots \\
b_{4 p 1}^{\mathrm{I}}+\hat{\ell}_{4 p 1}^{\mathrm{I}} b_{4 p 2}^{\mathrm{I}}+\widehat{\ell}_{4 p 2}^{\mathrm{I}} \cdots & b_{4 p r_{1}}^{\mathrm{I}}+\widehat{\ell}_{4 p r_{1}}^{\mathrm{I}} & \cdots & b_{4 p 4 p}^{\mathrm{I}}+\widehat{\ell}_{4 p 4 p}^{\mathrm{I}}
\end{array}\right]
$$

with $\mathrm{I}=1 \ldots N$. In writing these expressions the integer $p$ is assumed to be some fixed counting number. The integers $r_{1}$ to $r_{4 p}$ are allowed to range from 0 to $4 p-1$ and similarly the integers $s_{1}$ to $s_{4 p}$ are allowed to range from 0 to $4 p-1$. We also assume that the numerical values of all the entries in the matrices are such that they satisfy the constraints in satisfy the conditions in (7.5) - (7.8).

Next we imagine there is a sender who wishes to send an encrypted version of these to a receiver. The method of encryption is very simple. The encrypted versions transmitted in the open have all their $\ell$-parameters and $\widehat{\ell}$-parameters set to zero. From the examples we have worked out previously, we know in some cases (with a relatively small amount of 
effort) the receiver can set up calculations to reconstruct the encrypted matrices. What the examples do not show us is how general is this capability. We assert understanding this problem in its generality is equivalent to solving the adinkra network version of the auxiliary field problem. As cryptography is a very well developed topic, it may well be that this alternate formulation of the problem can take advantage of some of this preexisting knowledge.

\section{Summary and conclusion}

The most important result of this work is the demonstration that given the information of an on-shell adinkra network it is possible by use of the Garden Algebra to derive a corresponding off-shell structure in which the on-shell one is embedded.

The method we have introduced involves the introduction of a space of real parameters, denoted by $\ell$ 's, which are used to construct matrices that interpolate from a description of an on-shell adinkra network to an off-shell one. There may be an interesting mathematical question to pursue here. If we think of the $\ell$ 's as the coordinates of some space, then the solution to the "Garden Algebra" problem for augmented on-shell L-matrices and Rmatirces may be regarded as the search for the loci of points which simultaneously solve the conditions arising from the "Garden Algebra." This offers the possibility of attacking such problems from the point of view of real algebraic geometry. Within the DFGHILM [29] collaboration, but in unpublished private discussions, it has long been recognized that for some theories (with more than four colors), there exist the possibility that there not only exist isolated points that satisfy the "Garden Algebra" conditions, but entire surfaces.

Via adinkras and their adjacency matrices, the off-shell auxiliary field problem of supersymmetrical systems has been "translated" into more precise mathematical questions. The statement of these problems can be cast in the following form. Begin with a set of $\mathcal{N}$ $\mathrm{d}_{L} \times \mathrm{d}_{R}$ set of L-matrices and a set of $\mathcal{N} \mathrm{d}_{R} \times \mathrm{d}_{L}$ set of R-matrices. By the augmentation process described in the last section, these can be enlarged to be $4 p \times 4 p$ matrices for some integer $p$. Given an arbitrary set of the initial $\mathrm{d}_{L} \times \mathrm{d}_{R}$ and $\mathrm{d}_{R} \times \mathrm{d}_{L}$ matrices, is it possible to find augmentations that satisfy the conditions in (7.5) and (7.6)?

We have two conjectures to make along these lines.

Conjecture \# 1. In the work of [23], the L-matrices and R-matrices of a formulation of the $4 \mathrm{D}, \mathcal{N}=1$ double tensor adinkra network were given and this system does not possess an augmentation satisfying (7.5) and (7.6) in an irreducible manner.

Conjecture \# 2. In the work of [25], the L-matrices and R-matrices of a formulation of the $4 \mathrm{D}, \mathcal{N}=4$ Maxwell adinkra network were given and this system possesses an augmentation satisfying (7.5) and (7.6) in an irreducible manner.

With this work, we provided a proof of concept that the (R), (AD), and (I) steps are all implementable in the context of supersymmetrical field theories. However, even if one is successful in all of these, there remains a challenge that caution bids us to note. The $(\mathrm{O})$ operation, denoting the dimensional enhancement of the adinkra network world results, 
must then be converted first back into 0-brane world results and hence dimensionally enhanced back to a full Minkowskian space construction and this is not yet guaranteed to us. It could be that there exists some obstruction to carrying out this step even though all the other steps of the RADIO proposal are successful.

Though we are mindful of this possibility, we are also optimistic as in recent times, we have developed an understanding and powerful tools ("Adinkra/Gamma Matrix Equations," "Coxeter Group Orbit/Hodge Duality Relations," and "Holoraumy") [16, 17, 24, 30] which strongly suggest the existence of invariants that can be used to start from an adinkra network world description and recover a corresponding 0-brane world description. Once this is done, we believe the step of dimensional enhancement or $(\mathrm{O})$ "oxidation" should be straightforward.

"An error does not become truth by reason of multiplied propagation, nor does truth become error because nobody sees it. Truth stands, even if there be no public support. It is self sustained." - M. K. Ghandi

\section{Acknowledgments}

We would like to acknowledge Professors Kevin Iga, Tristan Hübsch, Kory Stiffler, and Stefan Mendez-Diaz for conversations. This work was partially supported by the National Science Foundation grant PHY-0354401 and in part by the University of Maryland Center for String \& Particle Theory. Additional acknowledgment is given by M. Calkins and D.E.A. Gates to the Center for String and Particle Theory, as well as recognition for their participation in 2013 \& 2014 SSTPRS (Student Summer Theoretical Physics Research Session) programs. The adinkras in this work were drawn with the aid of T. Hübsch. Finally, SJG wishes to thank J. H. Schwarz for the introduction to this problem and encouragement over decades.

Open Access. This article is distributed under the terms of the Creative Commons Attribution License (CC-BY 4.0), which permits any use, distribution and reproduction in any medium, provided the original author(s) and source are credited.

\section{References}

[1] W. Siegel and M. Roček, On off-shell supermultiplets, Phys. Lett. B 105 (1981) 275 [INSPIRE].

[2] S.J. Gates Jr. and S. Vashakidze, On $D=10, N=1$ Supersymmetry, Superspace Geometry and Superstring Effects, Nucl. Phys. B 291 (1987) 172 [INSPIRE].

[3] E. Bergshoeff, M. Rakowski and E. Sezgin, Higher derivative super Yang-Mills theories, Phys. Lett. B 185 (1987) 371 [INSPIRE].

[4] K. Peeters, P. Vanhove and A. Westerberg, Supersymmetric $R^{4}$ actions and quantum corrections to superspace torsion constraints, hep-th/0010182 [INSPIRE]. 
[5] M. Cederwall, B.E.W. Nilsson and D. Tsimpis, The Structure of maximally supersymmetric Yang-Mills theory: Constraining higher order corrections, JHEP 06 (2001) 034 [hep-th/0102009] [INSPIRE].

[6] S.F. Kerstan, Supersymmetric Born-Infeld from the D9-brane, Class. Quant. Grav. 19 (2002) 4525 [hep-th/0204225] [INSPIRE].

[7] N. Berkovits and V. Pershin, Supersymmetric Born-Infeld from the pure spinor formalism of the open superstring, JHEP 01 (2003) 023 [hep-th/0205154] [INSPIRE].

[8] S.J. Gates and L. Rana, A Theory of spinning particles for large- $N$ extended supersymmetry, Phys. Lett. B 352 (1995) 50 [hep-th/9504025] [INSPIRE].

[9] S.J. Gates Jr. and L. Rana, A Theory of spinning particles for large- $N$ extended supersymmetry. 2., Phys. Lett. B 369 (1996) 262 [hep-th/9510151] [INSPIRE].

[10] M. Faux and S.J. Gates Jr., Adinkras: A Graphical technology for supersymmetric representation theory, Phys. Rev. D 71 (2005) 065002 [hep-th/0408004] [INSPIRE].

[11] C.F. Doran et al., On graph-theoretic identifications of Adinkras, supersymmetry representations and superfields, Int. J. Mod. Phys. A 22 (2007) 869 [math-ph/0512016] [INSPIRE].

[12] C.F. Doran et al., Relating Doubly-Even Error-Correcting Codes, Graphs and Irreducible Representations of N-Extended Supersymmetry, arXiv:0806.0051 [INSPIRE].

[13] C.F. Doran et al., Codes and Supersymmetry in One Dimension, Adv. Theor. Math. Phys. 15 (2011) 1909 [arXiv:1108.4124] [INSPIRE].

[14] C.F. Doran et al., Topology Types of Adinkras and the Corresponding Representations of $N$-Extended Supersymmetry, arXiv:0806.0050 [INSPIRE].

[15] Y. Zhang, Adinkras for Mathematicians, Trans. Am. Math. Soc. 366 (2014) 3325.

[16] S.J. Gates Jr., The Search for Elementarity Among Off-Shell SUSY Representations, The Korean Institute for Advanced Studies (KIAS) Newsletter 5 (2012) 19.

[17] I. Chappell, Isaac, S.J. Gates and T. Hübsch, Adinkra (in)equivalence from Coxeter group representations: A case study, Int. J. Mod. Phys. A 29 (2014) 1450029 [arXiv:1210.0478] [INSPIRE].

[18] C. Doran, K. Iga, G. Landweber and S. Mendez-Diez, Geometrization of N-Extended 1-Dimensional Supersymmetry Algebras, arXiv:1311.3736 [INSPIRE].

[19] S.J. Gates Jr. and L. Rana, On Extended Supersymmetric Quantum Mechanics, UMDEPP 93-194 (1994), unpublished.

[20] S.J. Gates Jr. and L. Rana, Ultramultiplets: A New representation of rigid 2-d, $N=8$ supersymmetry, Phys. Lett. B 342 (1995) 132 [hep-th/9410150] [INSPIRE].

[21] S.J. Gates Jr. and L. Rana, Tuning the RADIO to the off-shell 2-D Fayet hypermultiplet problem, hep-th/9602072.

[22] S.J. Gates Jr., S. Randall and K. Stiffler, Reduction Redux of Adinkras, Int. J. Mod. Phys. A 29 (2014) 1450070 [arXiv: 1312 .2000] [INSPIRE].

[23] J. Gates et al., 4D, $N=1$ Supersymmetry Genomics (I), JHEP 12 (2009) 008 [arXiv:0902.3830] [INSPIRE]. 
[24] S.J. Gates, T. Hubsch and K. Stiffler, On Clifford-Algebraic "Holoraumy", Dimensional Extension and SUSY Holography, arXiv: 1409.4445 [INSPIRE].

[25] M. Calkins, D.E.A. Gates, S.J. Gates and B. McPeak, Is it possible to embed a $4 D, \mathcal{N}=4$ supersymmetric vector multiplet within a completely off-shell adinkra hologram?, JHEP 05 (2014) 057 [arXiv: 1402.5765] [INSPIRE].

[26] S.J. Gates Jr., J. Parker, V.G.J. Rodgers, L. Rodriguez and K. Stiffler, A Detailed Investigation of First and Second Order Supersymmetries for Off-Shell $N=2$ and $N=4$ Supermultiplets, arXiv:1106.5475 [INSPIRE].

[27] S.J. Gates and L. Rana, A Theory of spinning particles for large- $N$ extended supersymmetry, Phys. Lett. B 352 (1995) 50 [hep-th/9504025] [INSPIRE].

[28] S.J. Gates Jr. and L. Rana, A Theory of spinning particles for large- $N$ extended supersymmetry. 2., Phys. Lett. B 369 (1996) 262 [hep-th/9510151] [INSPIRE].

[29] http://math.bard.edu/DFGHIL/index.php?n=Main.Publications.

[30] M. Calkins, D.E.A. Gates, S.J. Gates and K. Stiffler, Adinkras, O-branes, Holoraumy and the SUSY QFT/QM Correspondence, arXiv:1501.00101 [INSPIRE]. 